\title{
Regularized Deconvolution Method for Turbulent Combustion Modeling
}

\author{
Qing Wang, Matthias Ihme* \\ Department of Mechanical Engineering, Stanford University
}

\begin{abstract}
The modeling of filtered chemical source terms in large-eddy simulation (LES) of turbulent reacting flows remains a challenge. Deconvolution methods are an attractive technique for representing these unclosed terms. With this technique, filtered scalars are reconstructed through deconvolution. The chemical source terms that are computed directly from the deconvolved scalars are filtered explicitly to represent the turbulence-chemistry interaction. However, the approximate deconvolution method (ADM), frequently employed for non-reacting flows, exhibits shortcomings for reacting scalars. This is because ADM does not ensure essential conservation and boundedness conditions. To address this issue, we propose a regularized deconvolution method (RDM) based on an optimization procedure. We conduct a priori and a posteriori analyses to examine RDM as a closure in LES. These investigations are performed in the context of explicit filtering. By showing that RDM is accurate and stable with respect to both the filter width and time, we conclude that the new deconvolution method shows promise in application as a combustion model for unclosed term in combustion LES.
\end{abstract}

Keywords: Large-eddy simulation; Turbulent combustion; Deconvolution; Regularization; Partially-premixed combustion

\section{Introduction}

Large-eddy simulation (LES) is now recognized as a viable method for predicting turbulent reacting flows [1]. In most problems involving turbulent reacting flows, the underlying physics is characterized by the dynamics of large scale flow structures and their interactions with the flame. In LES, large-scale structures are represented explicitly on the computational mesh. By modeling the subgrid scale (SGS) residual contributions, this approach improves descriptions of scalar mixing and turbulent chemistry interaction.

The evolution of reactive flows is described by the solution of the reactive Navier-Stokes equations. Defining $\phi(\boldsymbol{x}, t)$ as a generic reactive scalar, the corresponding transport equation is written as:

$$
\frac{\partial}{\partial t}(\rho \phi)+\frac{\partial}{\partial x_{i}}\left(\rho u_{i} \phi\right)=\frac{\partial}{\partial x_{i}}\left(\rho D_{\phi} \frac{\partial \phi}{\partial x_{i}}\right)+\rho \dot{\omega}_{\phi},
$$

where $t$ is the time, $x_{i}$ is the spacial coordinate along the $i$ th direction, $\rho$ is the density, $u_{i}$ is the $i$ th velocity component, $D_{\phi}$ is the molecular diffusivity of scalar $\phi$ and $\dot{\omega}_{\phi}$ is the source term of $\phi$ due to chemical reaction. Solving Eq. (1) provides information about $\phi$ at all scales. To achieve this, the minimum resolution requirement is constrained by the smallest characteristic length scale in the flow field. For instance, the length scale can be determined by the minimum among the Kolmogorov scale, Bachelor scale and the flame thickness, depending on the flow configuration. In LES, the mesh size is characterized by the length scale where the inertial range is resolved [2] instead of resolving the smallest physical length scale. In this approach, reactive scalars need to be filtered by a low-pass filter. Denoting $G(\boldsymbol{x})$ as the time-invariant filter

\footnotetext{
* Corresponding author

Email addresses: wangqing@stanford.edu (Qing Wang), mihme@stanford.edu (Matthias Ihme)
} 
kernel in physical space, satisfying the property $\int_{-\infty}^{\infty} G(\boldsymbol{x}) d \boldsymbol{x}=1$, the explicit filtering of $\phi(\boldsymbol{x}, t)$ is given by the following convolution operation:

$$
\bar{\phi}(\boldsymbol{x}, t)=\int_{-\infty}^{\infty} G\left(\boldsymbol{x}-\boldsymbol{x}^{\prime}\right) \phi\left(\boldsymbol{x}^{\prime}, t\right) d \boldsymbol{x}^{\prime}=: G * \phi
$$

Hence, the governing equation for a reactive scalar in LES is obtained by applying the low-pass filter $G$ to Eq. (1),

$$
\frac{\partial}{\partial t}(\bar{\rho} \widetilde{\phi})+\frac{\partial}{\partial x_{i}}\left(\bar{\rho} \widetilde{u_{i}} \widetilde{\phi}\right)=\overline{\frac{\partial}{\partial x_{i}}\left(\rho D \frac{\partial \phi}{\partial x_{i}}\right)}+\sigma_{\phi}+\widetilde{\rho} \widetilde{\dot{\omega}_{\phi}}
$$

where $\widetilde{\phi}$ is the Favre filtered scalar, which is defined as

$$
\widetilde{\phi}(\boldsymbol{x}, t)=\frac{\overline{\rho \phi}}{\bar{\rho}}=: \widetilde{G} * \phi
$$

Terms on the right hand side of Eq. (3) are unclosed; therefore modeling of these terms is required to solve the Favre-filtered LES equation for $\phi$. In this paper, we propose closure models for these unclosed terms using the deconvolution method. This approach addresses some of the issues we have in closing these terms, particularly for the filtered chemical source term. The issues are discussed below in this section.

The filtered molecular diffusion term appearing as the first term on the right-hand-side of Eq. (3) is commonly modeled by retaining first-order terms and neglecting higher order contributions. In general, the closure of this term does not require application of a deconvolution method and takes the following form:

$$
\overline{\frac{\partial}{\partial x_{i}}\left(\rho D_{\phi} \frac{\partial \phi}{\partial x_{i}}\right)}=\frac{\partial}{\partial x_{i}}\left(\bar{\rho} \widetilde{D_{\phi}} \frac{\partial \widetilde{\phi}}{\partial x_{i}}\right) .
$$

The second term we need to address is the turbulent scalar mixing term, $\sigma_{\phi}$, which is defined as

$$
\sigma_{\phi}=\frac{\partial}{\partial x_{i}}\left(\bar{\rho} \widetilde{u_{i}} \widetilde{\phi}\right)-\overline{\frac{\partial}{\partial x_{i}}\left(\rho u_{i} \phi\right)}
$$

A typical closure for this term is to use a gradient model [3], which introduces a turbulent diffusivity. A dynamic procedure for evaluating this term has been developed [4].

Modeling of the filtered chemical source term, appearing as last term on the right-hand-side of Eq. (3), is especially challenging. The difficulty arises for two reasons, namely the modeling of chemical reactions and the modeling of the turbulence-chemistry interaction on unfiltered scales. The chemical reactions are usually represented either using detailed or reduced chemistry in Arrhenius form, or relying on reactiontransport manifolds. Examples of combustion models that utilize Arrhenius laws are the artificially thickened flame model [5] and the linear-eddy model [6]. Some examples for manifold-based models are intrinsic low dimensional manifold (ILDM) [7], flame prolongation in ILDM [8], flamelet generated manifold [9], and flamelet progress variable [10]. An adaptive modeling approach that integrates different combustion submodels was developed by introducing a Pareto efficiency $[11,12]$. In these models, the thermochemical state is represented in terms of a reduced set of scalars. While these methods are effective in reducing the computational complexity of the simulation, they rely on intrinsic assumptions about the underlying flame structure representation and closure models for turbulence-chemistry interaction.

To consider effects of turbulence on the combustion process, a closure model is required. For Arrhenius chemistry, models are developed based on large-scale structures. An example for this type of model is the laminar chemistry method [13] which uses the resolved scalars to compute the reaction source term. Other examples are the partially stirred reactor (PaSR) model $[14,15]$ and the scale-similarity closure [16], which extends the laminar chemistry method by modeling the effects of fine-scale structures. In contrast, reactiontransport manifold models typically consider the asymptotic regimes of premixed and diffusion flames. Topology-dependent closures were developed for these models, such as the filtered tabulated chemistry model 
for LES [17] and presumed probability density function (PDF) models [18]. Although these models have been shown to provide reliable predictions of canonical and complex flame configurations, the dependence on an underlying flame topology limits their application to combustion problems involving a single-flame regime. Therefore, for multi-regime and mixed-mode combustion, submodel adaptation [11, 12] or the use of topology-free combustion models is required.

To address this need, turbulent closures using deconvolution methods are considered. Deconvolution methods that are developed from mathematical arguments provide approximate inverse to the filter operation in LES. Consequently, SGS terms in the LES equations can be computed explicitly using the deconvolved variables. Since no assumptions on the reconstructed flow field are made in deriving the deconvolution operator, this approach has the potential to be independent of an underlying flame topology and therefore applicable as subgrid model for both finite rate chemistry and reduced manifold models.

The deconvolution method was first introduced as a closure for the SGS stress terms in LES of nonreactive flows [19], and the method was applied to different flow configurations [20, 21, 22]. The application of the deconvolution method to reactive LES was later formulated using a moment-based reconstruction of the scalar field [23, 24, 25]. An approximate deconvolution operator derived from a Taylor-series expansion to the Gaussian filter was developed [26]. In conjunction with a flamelet model, this model was applied to a turbulent Bunsen flame.

However, by recognizing that deconvolution operators developed for LES are linear operators, they lack essential boundedness and conservation properties of scalar quantities, which are critical for turbulent reacting flows. By addressing this issue, the objective of this work is to propose a general closure for unclosed terms in Eq. (3) by developing a regularized deconvolution method (RDM). To this end, constraints are directly introduced in RDM to fulfill essential regularization conditions.

The remainder of this paper has the following structure. The governing equations are introduced in Sec. 2. Section 3 is concerned with reviewing mathematical formulations of classical deconvolution techniques and developing the regularized deconvolution method. We examine the deconvolution methods by considering DNS of a partially-premixed flame in canonically decaying turbulence. The computational configuration is described in Sec. 4. We first conduct an a priori study to quantify the accuracy of the deconvolution methods in terms of scalar reconstruction and the estimation of unclosed terms in Sec. 5. Then in Sec. 6 we analyze the performance of RDM in an a posteriori study. The paper concludes with an overall evaluation of RDM and its applications in LES in Sec. 7.

\section{Governing equations of LES}

In combustion LES, the generic scalar transport equation, shown in Eq. (3), has to be adapted for the temperature and species mass fractions $Y_{k}, k=1, \cdots, N_{s}$, with $N_{s}$ denoting the number of species. The full set of filtered equations for reactive flows in the low-Mach number limit takes the following form:

$$
\begin{aligned}
\frac{\partial}{\partial t} \bar{\rho}+\frac{\partial}{\partial x_{i}}\left(\bar{\rho} \widetilde{u}_{i}\right) & =0 \\
\frac{\partial}{\partial t}\left(\bar{\rho} \widetilde{u}_{j}\right)+\frac{\partial}{\partial x_{i}}\left(\bar{\rho} \widetilde{u_{i}} \widetilde{u_{j}}\right) & =-\frac{\partial \bar{p}}{\partial x_{j}}+\frac{\partial \overline{\tau_{i j}}}{\partial x_{i}}+\sigma_{u_{j}} \\
\frac{\partial}{\partial t}\left(\bar{\rho} \widetilde{Y_{k}}\right)+\frac{\partial}{\partial x_{i}}\left(\bar{\rho} \widetilde{u_{i}} \widetilde{Y_{k}}\right) & =\frac{\frac{\partial}{\partial x_{i}}\left(\rho D_{k} \frac{\partial Y_{k}}{\partial x_{i}}\right)}{}+\sigma_{Y_{k}}+\overline{\rho \dot{\omega}_{Y_{k}}} \\
\frac{\partial}{\partial t}(\bar{\rho} \widetilde{T})+\frac{\partial}{\partial x_{i}}\left(\bar{\rho} \widetilde{u_{i}} \widetilde{T}\right) & =\frac{\left.\frac{1}{C_{p}} \frac{\partial}{\partial x_{i}}\left(\lambda \frac{\partial T}{\partial x_{i}}\right)+\sigma_{T}+\frac{\rho \dot{\omega}_{T}^{\prime}}{C_{p}}\right)+}{\left(\rho \sum_{k=1}^{N_{s}} \frac{C_{p, k}}{C_{p}} D_{k} \frac{\partial Y_{k}}{\partial x_{i}}\right) \frac{\partial T}{\partial x_{i}}+\frac{1}{C_{p}} \frac{\partial p_{0}}{\partial t}}
\end{aligned}
$$

where $p$ is the hydrodynamic pressure, $C_{p}=\sum_{k=1}^{N_{s}} C_{p, k} Y_{k}$ is the heat capacity of the gas mixture, and $\tau_{i j}=\mu\left(\partial u_{i} / \partial x_{j}+\partial u_{j} / \partial x_{i}-2 / 3 \delta_{i j} \partial u_{k} / \partial x_{k}\right)$ is the viscous stress tensor. Note that Eq. (7d) is written for 


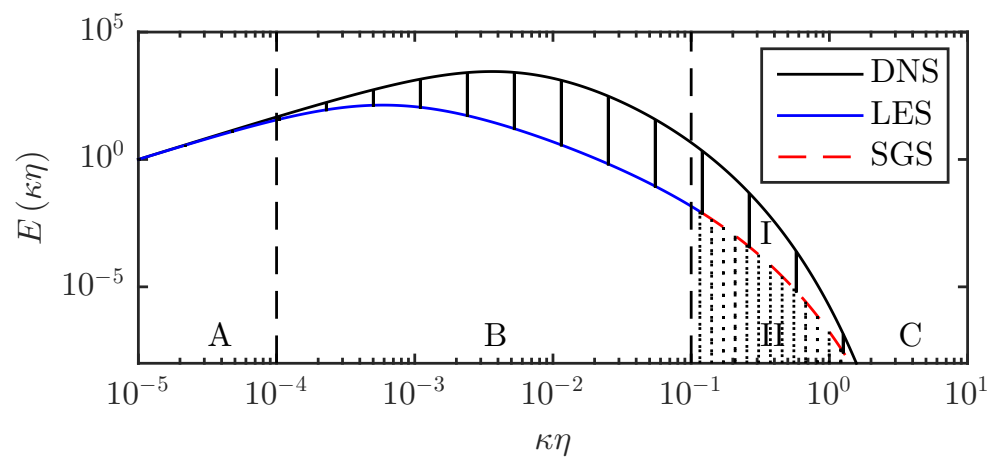

Figure 1: (Color online) Schematic of energy spectrum in the context of DNS, LES and modeling potentials.

low Mach number flow conditions, in which viscous-dissipative effects are ignored, and the thermodynamic pressure $p_{0}$ is only a function of time [27], which is evaluated from the state equation.

In this set of equations, Eqs. (7), the divergence of the Reynolds stress tensor $\sigma_{u_{j}}$, the turbulent mixing terms $\sigma_{\phi}$ for $\phi \in\{\boldsymbol{Y}, T\}$ are defined as

$$
\begin{aligned}
\sigma_{u_{j}} & =\frac{\partial}{\partial x_{i}}\left(\bar{\rho} \widetilde{u_{i}} \widetilde{u_{j}}\right)-\overline{\frac{\partial}{\partial x_{i}}\left(\rho u_{i} u_{j}\right)}, \\
\sigma_{\phi} & =\frac{\partial}{\partial x_{i}}\left(\bar{\rho} \widetilde{u_{i}} \widetilde{\phi}\right)-\overline{\frac{\partial}{\partial x_{i}}\left(\rho u_{i} \phi\right)} .
\end{aligned}
$$

The last terms on the right-hand-side of Eqs. (8) are unclosed, and must be modeled.

\section{Deconvolution methods}

Deconvolution methods are used to approximate unfiltered quantities in LES and as closure models for SGS terms. The principle of deconvolution methods in LES is schematically illustrated in Fig. 1 for a spectrally non-local filter. Comparing the energy spectrum of LES to DNS, we can divide the spectral behavior of LES into three regions. Region A corresponds to the fully resolved flow-field; in this region of the spectrum all length scales are represented by the mesh. Region B is the region where the solution field is under-resolved. In this region, although all length scales are represented by the mesh, discrepancies exist between LES and DNS due to the filtering. Region $\mathrm{C}$ is the region where the variable is not resolved. To represent the energy in region C, a SGS model is required for LES, as shown by the shaded area II. Note that the energy is still under-resolved for LES with SGS model due to the LES filtering, as shown by area I. To restore this portion of the energy, a deconvolution model is required to invert the LES-filter solution field. In this paper, we focus on investigating the deconvolution method that maximizes the reconstruction in area I. As such, this analysis closely represents an explicit filtering method in LES.

Following the definition of the filter operations, the deconvolution operation can be derived as follows. Denoting $Q$ as the deconvolution kernel in physical space, the deconvolution operation of the filtered quantity $\bar{\phi}(\boldsymbol{x}, t)$ is given as:

$$
\phi^{\star}(\boldsymbol{x}, t)=\int_{-\infty}^{\infty} Q\left(\boldsymbol{x}-\boldsymbol{x}^{\prime}\right) \bar{\phi}\left(\boldsymbol{x}^{\prime}, t\right) d \boldsymbol{x}^{\prime}=: Q * \bar{\phi},
$$

where $\phi^{\star}$ is a deconvolved scalar. Accordingly, the Favre deconvolution takes the form:

$$
\phi^{\widetilde{夭}}(\boldsymbol{x}, t)=\frac{Q *(\overline{\rho \phi})}{Q * \bar{\rho}}=: \widetilde{Q} * \widetilde{\phi},
$$


where the superscript notation $\widetilde{\star}$ is introduced to distinguish the deconvolution procedure for Favre filtered scalars from that for Reynolds filtered scalars.

The deconvolved quantity is an approximation of the corresponding unfiltered quantity. Depending on the filter type, we have $\phi \simeq \phi^{\star}$ for a Reynolds-filtered quantity and $\phi \simeq \phi^{\widetilde{\star}}$ for a Favre-filtered quantity. With the deconvolution model, the unclosed terms in Eqs. (8), and the chemical source term for a generic scalar $\phi \in\{\boldsymbol{Y}, T\}$ can be closed as:

$$
\begin{aligned}
& \sigma_{u_{j}}^{\widetilde{1}}=\frac{\partial}{\partial x_{i}}\left(\bar{\rho} \widetilde{u_{i}} \widetilde{u_{j}}\right)-\overline{\frac{\partial}{\partial x_{i}}\left(\rho u_{i}^{\widetilde{\star}} u_{j}^{\widetilde{k}}\right)}, \\
& \sigma_{\phi}^{\widetilde{\star}}=\frac{\partial}{\partial x_{i}}\left(\bar{\rho} \widetilde{u_{i}} \widetilde{\phi}\right)-\overline{\frac{\partial}{\partial x_{i}}\left(\rho u_{i}^{\widetilde{\star}} \phi^{\widetilde{\star}}\right)}, \\
& \overline{\rho \dot{\omega}_{\phi}^{\widetilde{\star}}}=\overline{\rho \dot{\omega}_{\phi}\left(\boldsymbol{Y}^{\widetilde{\star}}, T^{\widetilde{\star}}\right)} \text {. }
\end{aligned}
$$

Note that the chemical source term also introduces a dependence on the thermodynamic pressure, which can also be considered using the deconvolution method.

As an aside, the explicit representation of the turbulent scalar mixing term $\sigma_{\phi}$ eliminates the need for using the classical gradient model to represent the SGS terms. In the gradient model, only the dissipation of energy is considered. The deconvolution representation potentially considers backscatter effects. In addition, with the explicit representation of the SGS terms, the issue of the misalignment between the turbulent scalar mixing and the scalar gradient direction becomes moot.

In the context of deconvolution, the set of equations for reactive flows can then be represented as:

$$
\begin{aligned}
& \frac{\partial}{\partial t} \bar{\rho}+\frac{\partial}{\partial x_{i}}\left(\bar{\rho} \widetilde{u}_{i}\right)=0 \\
& \frac{\partial}{\partial t}\left(\bar{\rho} \widetilde{u}_{j}\right)+\frac{\partial}{\partial x_{i}}\left(\bar{\rho} \widetilde{u_{i}} \widetilde{u_{j}}\right)=-\frac{\partial \bar{p}}{\partial x_{j}}+\frac{\partial \overline{\tau_{i j}^{\widetilde{\star}}}}{\partial x_{i}}+\sigma_{u_{i}}^{\widetilde{\star}}, \\
& \frac{\partial}{\partial t}\left(\bar{\rho} \widetilde{Y_{k}}\right)+\frac{\partial}{\partial x_{i}}\left(\bar{\rho} \widetilde{u_{i}} \widetilde{Y_{k}}\right)=\overline{\frac{\partial}{\partial x_{i}}\left(\rho D_{k} \frac{\partial Y_{k}^{\widetilde{\star}}}{\partial x_{i}}\right)}+\sigma_{Y_{k}}^{\widetilde{\rho}}+\overline{\rho \dot{\omega}_{Y_{k}}^{\widetilde{\star}}} \\
& \frac{\partial}{\partial t}(\bar{\rho} \widetilde{T})+\frac{\partial}{\partial x_{i}}\left(\bar{\rho} \widetilde{u_{i}} \widetilde{T}\right)=\overline{\frac{1}{C_{p}} \frac{\partial}{\partial x_{i}}\left(\lambda \frac{\partial T^{\widetilde{\star}}}{\partial x_{i}}\right)}+\overline{\sigma_{T}^{\widetilde{\star}}}+\overline{\left(\frac{\rho \dot{\omega}_{T}^{\prime \widetilde{\star}}}{C_{p}}\right)}+
\end{aligned}
$$

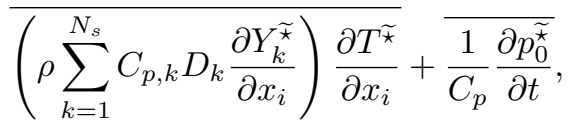

where the viscous stress tensor is computed as:

$$
\tau_{i j}^{\widetilde{\star}}=\mu\left(\frac{\partial u_{i}^{\widetilde{\star}}}{\partial x_{j}}+\frac{\partial u_{j}^{\widetilde{\star}}}{\partial x_{i}}-\frac{2}{3} \delta_{i j} \frac{\partial u_{k}^{\widetilde{\star}}}{\partial x_{k}}\right) .
$$

All thermo-viscous-diffusive transport properties and thermodynamic pressure are evaluated from deconvoluted quantities.

The realization of the deconvolution model is achieved through two different approaches, namely the approximate deconvolution method (ADM) and the Wiener filter. The latter forms the basis of RDM.

\subsection{Approximate deconvolution method (ADM)}

ADM [19] was derived from the van Cittert filter [28], which is defined by an iterative expression:

$$
\phi^{(\nu+1)}=\phi^{(\nu)}+\left(\bar{\phi}-G * \phi^{(\nu)}\right)
$$




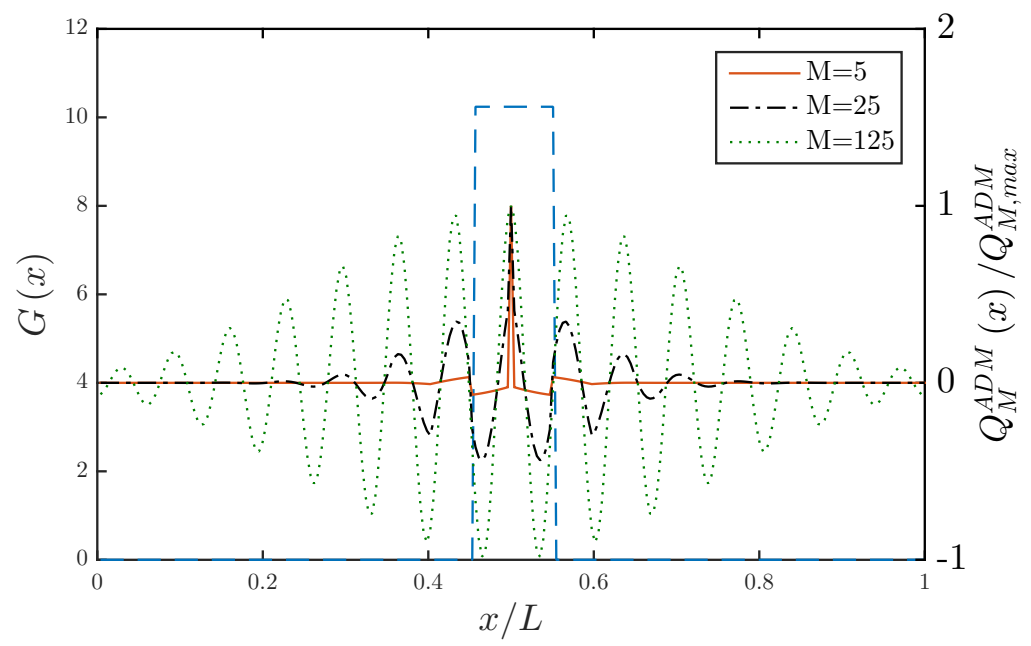

Figure 2: (Color online) Top-hat filter (dash line) and the ADM deconvolution operator with $M=\{5,25,125\}$ for filter width of $\Delta / L=0.1$ at $x_{0}=0.5$ in physical space.

where $\nu$ is the number of iterations. With this, the deconvolution operator can be derived recursively from the van Cittert filter and written in an explicit form as:

$$
Q_{M}^{\mathrm{ADM}}:=\sum_{\nu=0}^{M}(I-G)^{\nu} .
$$

In practice, the geometric series in Eq. (15) is truncated at a finite $M$. The ADM operator shown in Eq. (15) was analyzed in detail in [28] for non-reacting LES. Figure 2 shows an example of the 1D ADM operator with terms expanded explicitly to $M=\{5,25,125\}$. The test filter in Fig. 2 is a top-hat filter with filter width $\Delta / L=0.1$ in physical space. This filter is defined as:

$$
G\left(x, x_{0} ; \Delta\right)=\left\{\begin{array}{ll}
1 / \Delta & \left|x-x_{0}\right| \leq \Delta / 2 \\
0 & \text { otherwise }
\end{array} .\right.
$$

In Fig. 2, 250 grid points are used and the filter width is 25 grid points. Note that the operator for $M=5$ is bandwidth limited. With increasing $M, Q_{M}$ converges to the exact inverse of the filter operator $G$, in which case the width of $Q_{M}$ increases and eventually covers the entire computational domain. In addition, the amplitude of $Q_{M}$ grows unbounded. In Fig. 2, the peak values for $Q_{M, \max }$ with $M=\{5,25,125\}$ are $5.89,52.56$ and $4.08 \times 10^{9}$, respectively. In practice, $M=5$ is used in LES [19].

\subsection{Wiener filter}

A different approach to deconvolution is to use the Wiener filter [29], which is the solution to the minimum mean square error (MMSE) optimization problem. The MMSE problem minimizes the expectation of the difference between a generic scalar $\phi \in\{\boldsymbol{Y}, T\}$ and the deconvolved scalar $\phi^{\star}$ in Fourier space, which can be written as:

$$
\min _{\widehat{\phi}^{\star}(\boldsymbol{\kappa}, t)} \epsilon(\boldsymbol{\kappa}, t)=\mathrm{E}\left\{\left|\widehat{\phi}(\boldsymbol{\kappa}, t)-\widehat{\phi}^{\star}(\boldsymbol{\kappa}, t)\right|^{2}\right\}
$$

where E denotes the expectation operation, $\widehat{\phi}$ is the scalar in Fourier space, and $\widehat{\phi}^{\star}(\boldsymbol{\kappa}, t)=\widehat{Q}(\boldsymbol{\kappa}) \overline{\widehat{\phi}}(\boldsymbol{\kappa}, t)$ is the deconvolved scalar. Assuming that the filtered signal takes the form $\overline{\widehat{\phi}}(\boldsymbol{\kappa}, t)=\widehat{G}(\boldsymbol{\kappa}) \widehat{\phi}(\boldsymbol{\kappa}, t)+\widehat{n}(\boldsymbol{\kappa}, t)$, 
where $\widehat{n}(\boldsymbol{\kappa}, t)$ is the noise up to the order of the numerical scheme used in the simulation, the mean square error $\epsilon(\boldsymbol{\kappa}, t)$ can be expressed explicitly as:

$$
\begin{aligned}
\epsilon(\boldsymbol{\kappa}, t)= & |I-\widehat{Q}(\boldsymbol{\kappa}) \widehat{G}(\boldsymbol{\kappa})|^{2} \mathrm{E}\left\{|\widehat{\phi}(\boldsymbol{\kappa}, t)|^{2}\right\} \\
& -(I-\widehat{Q}(\boldsymbol{\kappa}) \widehat{G}(\boldsymbol{\kappa})) \widehat{Q}^{*}(\boldsymbol{\kappa}) \mathrm{E}\left\{\widehat{\phi}(\boldsymbol{\kappa}, t) \widehat{n}^{*}(\boldsymbol{\kappa}, t)\right\} \\
& -(I-\widehat{Q}(\boldsymbol{\kappa}) \widehat{G}(\boldsymbol{\kappa}))^{*} \widehat{Q}(\boldsymbol{\kappa}) \mathrm{E}\left\{\widehat{\phi}^{*}(\boldsymbol{\kappa}, t) \widehat{n}(\boldsymbol{\kappa}, t)\right\} \\
& +|\widehat{Q}(\boldsymbol{\kappa})|^{2} \mathrm{E}\left\{|\widehat{n}(\boldsymbol{\kappa}, t)|^{2}\right\},
\end{aligned}
$$

where the superscript $*$ denotes the complex conjugate of the corresponding quantity. Because $\widehat{\phi}(\boldsymbol{\kappa}, t)$ and $\widehat{n}(\boldsymbol{\kappa}, t)$ are independent samples, $\mathrm{E}\left\{\widehat{\phi}(\boldsymbol{\kappa}, t) \widehat{n}^{*}(\boldsymbol{\kappa}, t)\right\}$ and $\mathrm{E}\left\{\widehat{\phi}^{*}(\boldsymbol{\kappa}, t) \widehat{n}(\boldsymbol{\kappa}, t)\right\}$ are zero. By defining the ensemble averages of the power density function of the signal and noise as

$$
\begin{aligned}
& \widehat{S}(\boldsymbol{\kappa}, t)=\mathrm{E}\left\{|\widehat{\phi}(\boldsymbol{\kappa}, t)|^{2}\right\}, \\
& \widehat{N}(\boldsymbol{\kappa}, t)=\mathrm{E}\left\{|\widehat{n}(\boldsymbol{\kappa}, t)|^{2}\right\},
\end{aligned}
$$

Eq. (18) can be rewritten in the form

$$
\epsilon(\boldsymbol{\kappa}, t)=(I-\widehat{Q}(\boldsymbol{\kappa}) \widehat{G}(\boldsymbol{\kappa}))^{*}(I-\widehat{Q}(\boldsymbol{\kappa}) \widehat{G}(\boldsymbol{\kappa})) \widehat{S}(\boldsymbol{\kappa}, t)+\widehat{Q}(\boldsymbol{\kappa})^{*} \widehat{Q}(\boldsymbol{\kappa}) \widehat{N}(\boldsymbol{\kappa}, t) .
$$

Upon taking the derivative of $\epsilon(\boldsymbol{\kappa}, t)$ with respect to $\widehat{Q}(\boldsymbol{\kappa})$ and equating it to zero, we obtain the following solution to the MMSE problem:

$$
\widehat{Q}^{\mathrm{W}}(\boldsymbol{\kappa})=\frac{\widehat{G}^{*}(\boldsymbol{\kappa}) \widehat{S}(\boldsymbol{\kappa}, t)}{|\widehat{G}(\boldsymbol{\kappa})|^{2} \widehat{S}(\boldsymbol{\kappa}, t)+\widehat{N}(\boldsymbol{\kappa}, t)},
$$

where $\widehat{Q}^{\mathrm{W}}$ denotes the Wiener deconvolution. For a non-zero signal, $\widehat{S}(\boldsymbol{\kappa}, t)$ in Eq. (21) can be eliminated from both the numerator and the denominator, which results in the following form of the deconvolution model:

$$
\widehat{Q}^{\mathrm{W}}(\boldsymbol{\kappa})=\frac{\widehat{G}^{*}(\boldsymbol{\kappa})}{|\widehat{G}(\boldsymbol{\kappa})|^{2}+\widehat{\eta}(\boldsymbol{\kappa}, t)},
$$

where $\widehat{\eta}(\boldsymbol{\kappa}, t)=\widehat{N}(\boldsymbol{\kappa}, t) / \widehat{S}(\boldsymbol{\kappa}, t)$ is the inverse of the signal-to-noise ratio, and $\widehat{\eta}$ can be estimated based on knowledge of the spectral property of the problem and the numerical scheme used in the simulation. In the current study, we adopt a constant value of $\widehat{\eta}(\boldsymbol{\kappa}, t)=10^{-6}$.

In LES, filtering and deconvolution are commonly conducted in physical space. Therefore the Wiener filter is converted back to the physical domain through an inverse Fourier transform,

$$
Q^{\mathrm{W}}(\boldsymbol{x})=\mathcal{F}^{-1}\left(\widehat{Q}^{\mathrm{W}}(\boldsymbol{\kappa})\right) \text {. }
$$

Figure 3 illustrates the deconvolution operator for the top-hat filter function that was also represented in Fig. 2 in physical space. Note that the bandwidth of the Wiener filter is the same as the exact filter inversion. However, the amplitude of the Wiener filter is bounded, so that the inversion operation is well defined. As $\widehat{\eta} \rightarrow 0, Q^{\mathrm{W}}$ converges to the exact inverse of the filter.

In summary, both ADM and Wiener filter provide approximate inversions to a filter operator. The stencil width of the ADM operator is smaller than the Wiener filter; however, the Wiener filter provides better signal reconstruction than ADM. Note that both ADM and the Wiener filter are linear operators; they therefore produce limited control over the behavior of the deconvolved solutions, such as the boundedness and conservation. These issues will be addressed with the regularized deconvolution method, which is discussed next. 


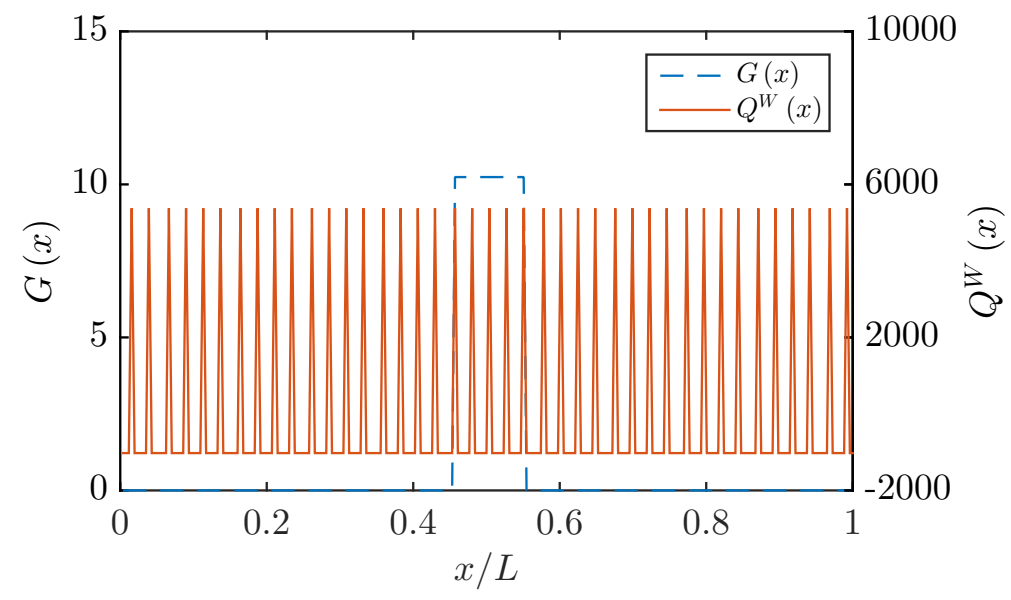

Figure 3: (Color online) Top-hat filter (dashed line) and the Wiener filter (solid line) for filter width of $\Delta / L=0.1$ at $x_{0}=0.5$ in physical space.

\subsection{Regularized deconvolution method (RDM)}

To apply deconvolution methods to the reactive scalars and temperature in Eqs. (7c) and (7d), we need to enforce the boundedness and conservation of these scalars. However, because both ADM and Wiener filter are linear operators, neither of these properties is ensured. To address the issue of boundedness, we reformulate the MMSE problem in Fourier space to a rank-deficient least-square problem in physical space, in which inequality constraints are enforced on a scalar $\phi \in\{\boldsymbol{Y}, T\}$. The resulting constraint can be written as:

$$
\begin{array}{ll}
\underset{\phi^{\star}(\boldsymbol{x}, t)}{\operatorname{minimize}} & \left\|\bar{\phi}(\boldsymbol{x}, t)-G(\boldsymbol{x}) * \phi^{\star}(\boldsymbol{x}, t)\right\|_{2}^{2} \\
\text { subject to } & \phi^{-} \leq \phi^{\star} \leq \phi^{+},
\end{array}
$$

where $\|\cdot\|_{2}$ is the $L_{2}$-norm and $\phi^{-}$and $\phi^{+}$correspond to the lower and upper limits of the scalar.

The optimization problem in Eq. (24) represents a quadratic programming problem with linear inequality constraints, which can be solved iteratively using active set method [30]. Denoting $\boldsymbol{\Phi}$ as the vector of scalar $\phi$ along one direction and $\boldsymbol{G}$ as the deconvolution kernel in matrix form, we can formulate the objective function in Eq. (24) by a linear system of equations as

$$
F\left(\boldsymbol{\Phi}^{\star}\right)=\boldsymbol{\Phi}^{\star T} \boldsymbol{H} \boldsymbol{\Phi}^{\star}-2 \widetilde{\boldsymbol{\Phi}}^{T} \boldsymbol{G} \boldsymbol{\Phi}^{\star}+\widetilde{\boldsymbol{\Phi}}^{T} \widetilde{\boldsymbol{\Phi}}
$$

where $\boldsymbol{H}=\boldsymbol{G}^{T} \boldsymbol{G}$ is the Hessian matrix of this problem. In each iteration, $\boldsymbol{\Phi}^{\star}$ is solved using a line-search method [30]. In particular, at iteration $k$, the line-search method updates $\boldsymbol{\Phi}_{k}^{\star}$ as

$$
\boldsymbol{\Phi}_{k}^{\star}=\boldsymbol{\Phi}_{k-1}^{\star}+\beta_{k} \boldsymbol{p}_{k},
$$

where $\beta_{k}$ is the step size and $\boldsymbol{p}_{k}$ is the search direction, which can be computed using Newton's method:

$$
\boldsymbol{H}_{k} \boldsymbol{p}_{k}=\nabla F\left(\boldsymbol{\Phi}_{k-1}^{\star}\right) .
$$

Note that $\boldsymbol{G}$ is a low rank matrix, consequently $\boldsymbol{H}$ in Eq. (25) is ill-conditioned and positive semi-definite. For indefinite $\boldsymbol{H}_{k}$, we can apply modified Cholesky factorization, which replaces the non-positive elements in the diagonal of the factorized matrix with small positive numbers.

In the active set method, we update the Hessian matrix $\boldsymbol{H}_{k}$ at each iteration. Specifically, we initialize $\boldsymbol{H}_{0}$ with $\boldsymbol{H}$ and $\boldsymbol{\Phi}_{0}^{\star}=\widetilde{\boldsymbol{\Phi}}$. Because $\widetilde{\phi}$ is bounded, $\widetilde{\boldsymbol{\Phi}}$ is within the feasible region of the solutions. In iteration 
$k$, inequality constraints that are violated in the line-search process in iteration $k-1$ are considered as active. These constraints are hence converted to equality constraints and are used to replace the inequality constraints in Eq. (24). In addition, we compute Lagrange multipliers for all currently included constraints. Those corresponding to negative Lagrange multipliers are considered as inactive. The Hessian matrix $\boldsymbol{H}_{k}$ is updated by appending the active constraints to $\boldsymbol{H}_{k-1}$ and removing the inactive constraints from it. The active set method proceeds until the optimality conditions are satisfied.

Another issue to be addressed in the deconvolution model is the property of conservation for reactive species. Instead of enforcing local mass conservation point-wise, global conservation for the mass fractions of the deconvolved scalars is enforced. This allows us to introduce this condition in Eq. (24) in the form of a Lagrangian multiplier, which can be solved computationally more efficiently. The resulting condition can then be written as:

$$
\underset{\boldsymbol{Y}^{\star}}{\operatorname{minimize}} \Psi\left(\boldsymbol{Y}^{\star}\right)=\int_{\Omega}\left|\sum_{k=1}^{N_{s}} Y_{k}^{\star}-1\right| d \boldsymbol{x},
$$

where $\Omega$ is the computational domain. Note that point-wise mass conservation is satisfied for the filtered reactive scalars, $\sum_{k=1}^{N_{s}} \widetilde{Y_{k}}=1$. With this, the objective function of Eq. (28) can be rewritten as:

$$
\Psi\left(\boldsymbol{Y}^{\star}\right)=\int_{\Omega}\left|\sum_{k=1}^{N_{s}}\left(Y_{k}^{\star}-\widetilde{Y_{k}}\right)\right| d \boldsymbol{x} .
$$

With the triangular inequality, the sequence of summation and integration can be interchanged to obtain an upper bound for the objective function:

$$
\Psi\left(\boldsymbol{Y}^{\star}\right) \leq \sum_{k=1}^{N_{s}}\left\|Y_{k}^{\star}-\widetilde{Y_{k}}\right\|_{1} .
$$

The minimization of Eq. (30) can be simplified by enforcing the optimal condition for each species, which is expressed as:

$$
\underset{Y_{k}^{\star}}{\operatorname{minimize}}\left\|Y_{k}^{\star}-\widetilde{Y_{k}}\right\|_{1}
$$

Finally, note that the $L_{1}$-norm is favorable for sparse data whereas scalar fields are typically non-sparse; therefore we replace the $L_{1}$-norm by the $L_{2}$-norm based on norm equivalence. This step has the added benefit of penalizing the solution toward large deviations. With this, the global conservation of the reactive scalar can be enforced by solving the following problem:

$$
\underset{Y_{k}^{\star}}{\operatorname{minimize}}\left\|Y_{k}^{\star}-\widetilde{Y_{k}}\right\|_{2}^{2}
$$

As an aside, imposing the local mass fraction conservation in deriving Eq. (32) weakly implies local conservation of the deconvolved reactive scalars $\boldsymbol{Y}^{\star}$. To further improve the accuracy in terms of mass fraction conservation, direct enforcement of local mass conservation can be done. This, however, requires an iterative procedure, which is computationally more expensive.

Equation (32) is embedded in Eq. (24) to form an augmented optimization problem for the reactive scalars, which is represented in the form:

$$
\begin{array}{ll}
\underset{Y_{k}^{\star}}{\operatorname{minimize}} & \left\|\widetilde{Y_{k}}-\widetilde{G} * Y_{k}^{\star}\right\|_{2}^{2}+\alpha\left\|Y_{k}^{\star}-\widetilde{Y_{k}}\right\|_{2}^{2} \\
\text { subject to } & Y_{k}^{-} \leq Y_{k}^{\star} \leq Y_{k}^{+}
\end{array}
$$

where $\alpha$ is the regularization parameter. In this study, $\alpha=0.01$ is used. By augmenting the optimization problem of the original deconvolution framework with the regularization of global scalar conservation, and enforcing physical constraints on the deconvolved scalars, the Wiener filter of Eq. (23) and Eq. (33) constitute 
RDM for generic scalars and species mass fractions where boundedness and conservation of these scalars are considered.

In the following, we develop a closure model for LES using RDM in conjunction with the framework of the Wiener filter. It was found that applying a directional decomposition of RDM, the accuracy of the deconvolution method is further improved since constraints on conservation and boundedness are applied along each spatial direction. The resulting algorithm is shown in Alg. 1. The deconvolution is first conducted using the Wiener filter $Q^{\mathrm{W}}$. Subsequently, boundedness and conservation are enforced. For this, regions in which the boundedness is violated are grouped into a subset. Equations (24) and (33) are then applied to temperature and species mass fractions in this subset. The initially deconvolved scalars are then updated with the corresponding results from RDM. This process is repeated for each spatial direction. With this approach, the scalars are regularized before the deconvolution iteration in the next direction. As a consequence, the size of the scalar subset that violates conservation and boundedness conditions is smaller than the size of the subset to which RDM is applied to after performing Wiener deconvolution in all directions.

As shown in Alg. 1, the RDM framework consists of two parts: one is to apply Wiener filtering and the other is to solve the optimization problem on a subset of the data. For the first part of this framework, the cost of the deconvolution is $\mathcal{O}\left(c N^{3}\right)$ where $c$ is the stencil size of the deconvolution operator, and $N$ is the number of grid points. This is the same as ADM since both methods are convolution operations in physical space. The cost of the second part of this framework depends on the size of the subset of data and the rate of convergence of the active set method. In our experience, the size of the subset is usually small because the Wiener filtering provides deconvolved solutions with high accuracy. Thus from our current experience, the cost of solving the optimization problem is negligible compared to the deconvolution.

\section{DNS configuration}

In the remainder of this work, we examine the performance of RDM. For this, we consider direct numerical simulations of a partially premixed flame in decaying turbulence. The computational setup is schematically shown in Fig. 4(a). The configuration consists of a cubic box with length $L=10 \mathrm{~mm}$ and 256 mesh points along each direction. Periodic boundary conditions are applied in each direction. The turbulent flow field is initialized using the von Karman-Pao (vKP) energy spectrum with a turbulent Reynolds number of $R e_{t}=$ 100 , and a Kolmogorov length scale of $\eta=0.2 \mathrm{~mm}$. The energy spectrum for velocity obtained numerically based on the analytic vKP profile at initial condition is shown in Fig. 5. In the current configuration, the initial eddy-turnover time scale is $\tau_{u}=1 \mathrm{~ms}$, which is used as reference for non-dimensionalization.

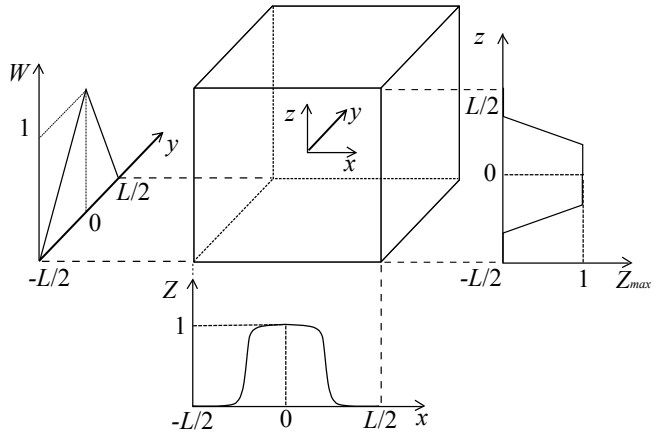

(a) DNS configuration with initialization of scalar profiles

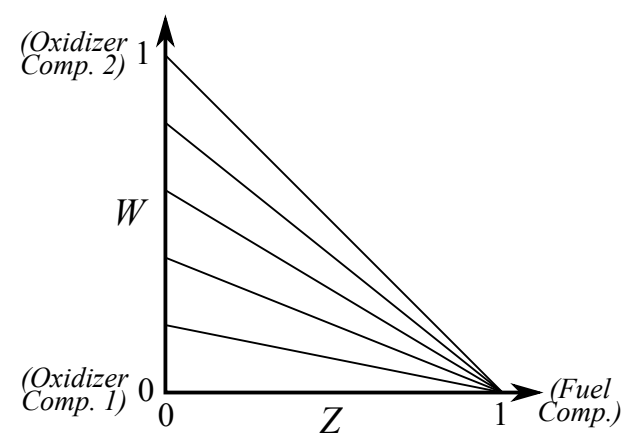

(b) Representation in composition space

Figure 4: DNS configuration of a partially premixed flame: (a) geometry with scalar initialization profile; (b) three-stream mixture fraction and definition of oxidizer split. 

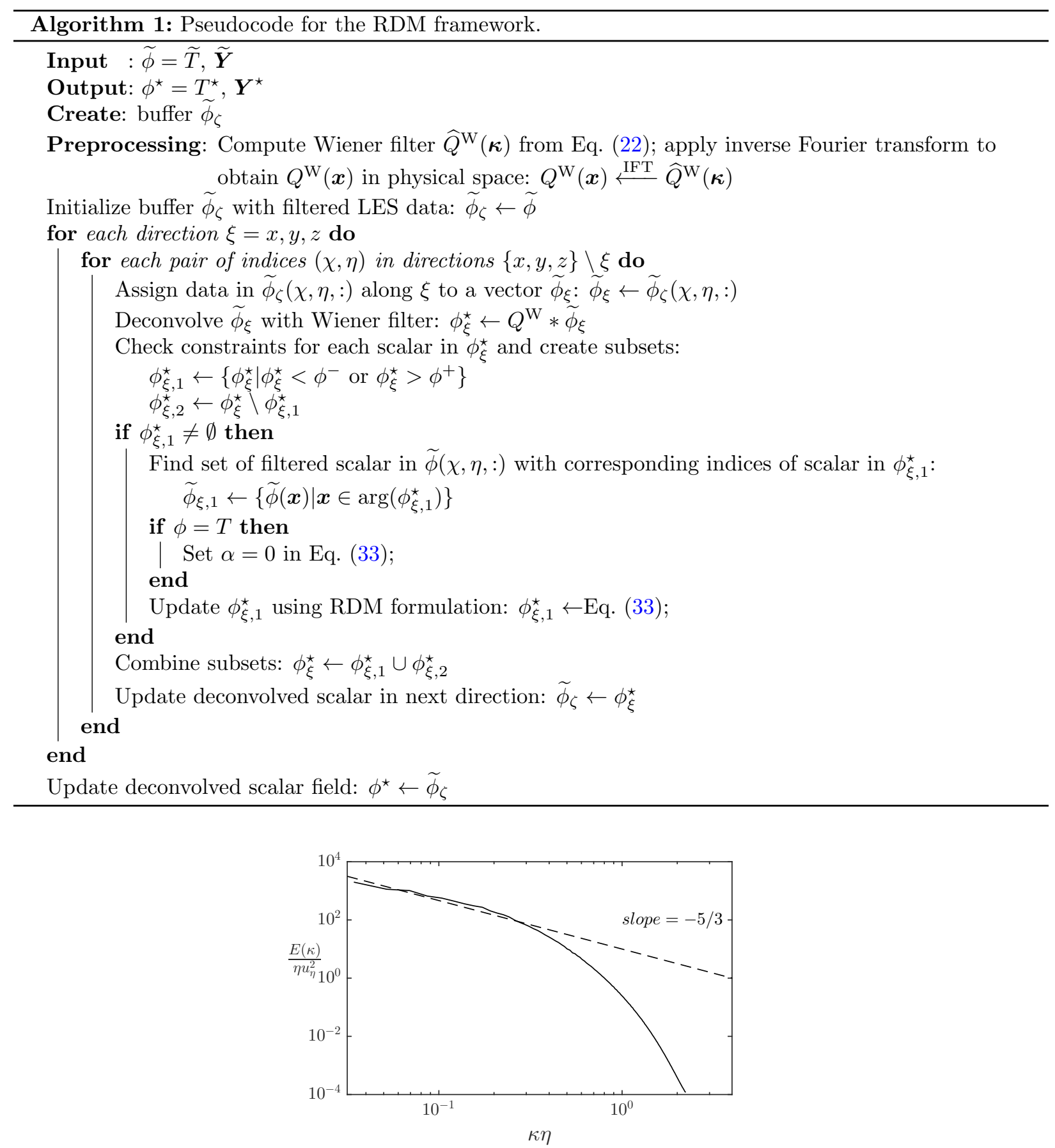

Figure 5: Energy spectrum at initial time step for velocity sampled using the vKP analytic profile.

The reaction chemistry is described by the following two-step skeletal methane mechanism [31, 32]:

$$
\begin{aligned}
\mathrm{CH}_{4}+1.5 \mathrm{O}_{2} & \rightarrow \mathrm{CO}+2 \mathrm{H}_{2} \mathrm{O}, \\
\mathrm{CO}+0.5 \mathrm{O}_{2} & \rightleftharpoons \mathrm{CO}_{2} .
\end{aligned}
$$

The reaction rates for the three elemental reactions are evaluated in Arrhenius form, using the following 
rate coefficients in cgs-units [31, 32]:

$$
\begin{aligned}
\dot{\omega}_{\mathrm{I}} & =10.0^{13.2} \exp (-24358 / T)\left[\mathrm{CH}_{4}\right]^{0.7}\left[\mathrm{O}_{2}\right]^{0.8}, \\
\dot{\omega}_{\text {IIf }} & =10.0^{14.6} \exp (-20131 / T)[\mathrm{CO}]\left[\mathrm{O}_{2}\right]^{0.25}\left[\mathrm{H}_{2} \mathrm{O}\right]^{0.5}, \\
\dot{\omega}_{\text {IIr }} & =5.0^{8.0} \exp (-20131 / T)\left[\mathrm{CO}_{2}\right],
\end{aligned}
$$

with $[\mathrm{A}]=\rho Y_{\mathrm{A}} / W_{\mathrm{A}}$ being the molar concentration of species $\mathrm{A}$.

To test the performance of the deconvolution methods in different combustion regimes, we construct a partially premixed flame configuration. The initial scalar fields are obtained from solutions of a three-stream flamelet model [33] that is shown in Fig. 4(b). In this model, $Z$ refers to the mixture fraction and $W$ is the oxidizer split. The initial profiles for these two scalars are given as follows:

$$
\begin{aligned}
Z & =\frac{1}{2}\left(1-\tanh \left[\frac{|x / L|-1 / 4}{\delta / L}\right]\right) \\
W & =1-2|y / L|, \\
Z_{\max } & = \begin{cases}1, & \text { if }|z / L| \leq 0.1 \\
1-2(10|z / L|-1), & \text { if } 0.10<|z / L|<0.15 \\
0, & \text { if }|z / L| \geq 0.15\end{cases}
\end{aligned}
$$

where $\delta$ is the flame thickness. The initial species and temperature fields are obtained by mapping the profile of $Z$ and $W$ in Fig. 4(a) on the flamelet solutions. With this definition, we construct the oxidizer composition at $Z=0$ using an inhomogeneous mixture of two conditions. The state at $W=0, Z=0$ has a mixture composition of air, with $Y_{\mathrm{O}_{2}}=0.23, Y_{\mathrm{N}_{2}}=0.77$ and $T=300 \mathrm{~K}$. The state at $W=1, Z=0$ has a mixture composition with $Y_{\mathrm{O}_{2}}=0.09, Y_{\mathrm{N}_{2}}=0.91$ and $T=1400 \mathrm{~K}$. And the state $W=0, Z=1$ refers to the fuel stream, consisting of pure methane at $T=300 \mathrm{~K}$. Adopting this configuration prevents us from limiting our scope to the asymptotic limits of premixed and diffusion combustion modes; instead, with stratification in the oxidizer composition and mixture fraction, we create a complex combustion environment to challenge the performance of the deconvolution methods.

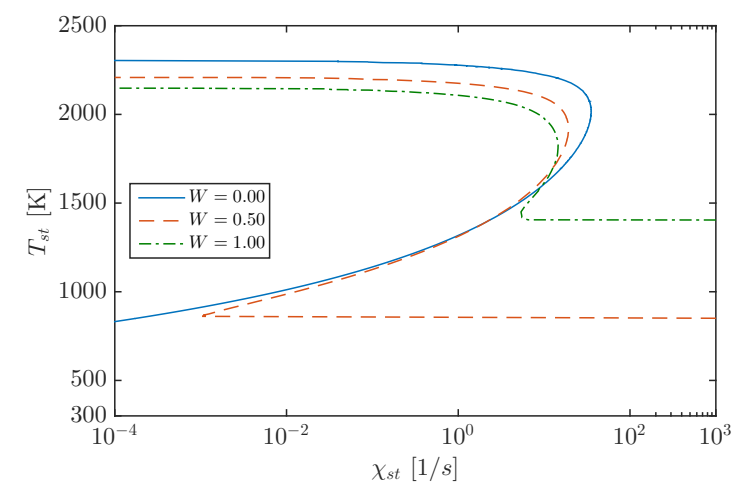

(a)

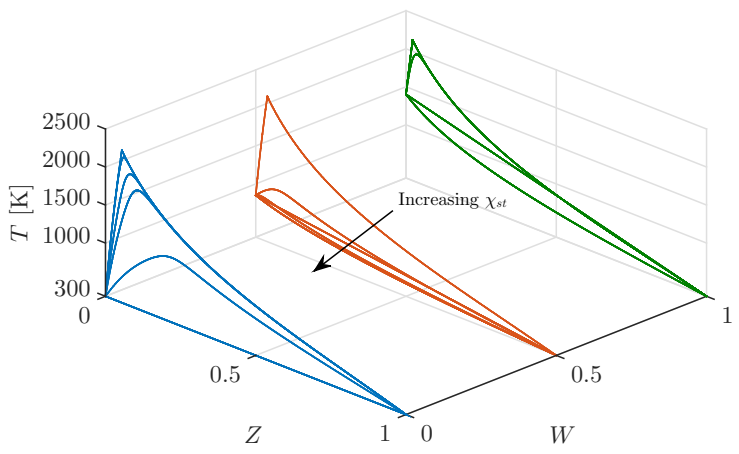

(b)

Figure 6: (Color online) Representative conditions of the chemical composition states used to initialize the DNS (a) S-shaped curve and (b) flamelet solutions for different values of oxidizer split, $W=\{0,0.5,1\}$.

Figure 6(a) shows the S-shaped curves of the partially premixed flame for three different oxidizer compositions, and Fig. 6(b) shows representative flamelet profiles. In this configuration, the igniting dissipation rate increases while the quenching dissipation rate reduces slightly as the parameter of the oxidizer split increases. To account for the effect of local extinction and re-ignition, scalars are initialized from flamelet solutions at $\chi_{s t}=5 \mathrm{~s}^{-1}$, which is close to the extinction point for all values of the oxidizer split. 
The shear layer thickness $\delta_{s}$ in this setup is evaluated as $1 \mathrm{~mm}$, according to the definition:

$$
\delta_{s}=\left(\frac{1}{|\nabla Z|}\right)_{Z=Z_{s t}} .
$$

This quantity will be used to non-dimensionalize the filter width.

Figure 7(a) shows the temporal evolution of turbulent kinetic energy (TKE) and the turbulent dissipation rate $\varepsilon$. We observe a high turbulent dissipation rate at the initial stage of the simulation, which rapidly decreases over the first two eddy-turnover times. As a consequence, a fast decay in TKE occurs over the first two eddy-turnover times, where a $90 \%$ reduction is observed. Figures 7 (b) and 7 (c) show the temporal evolution of mean temperature and mean species mass fractions profiles. Here, the mean value is denoted by angular brackets, $\langle\cdot\rangle$, and is evaluated from spatial averaging over the domain. We see that the flame approaches equilibrium conditions after around four eddy-turnover times. The peak chemical reaction rate, shown in Fig. 7(d), is observed at around three eddy-turnover times, and decreases by approximately $80 \%$ over the following eddy-turnover time. We observe a mild decay in chemical-reaction rate afterwards.

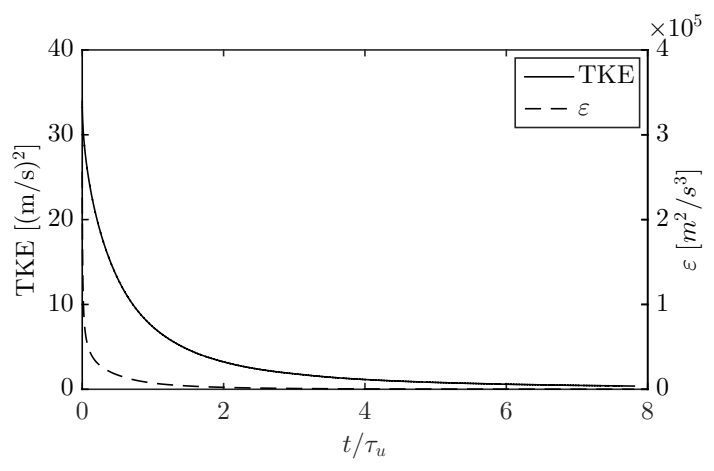

(a)

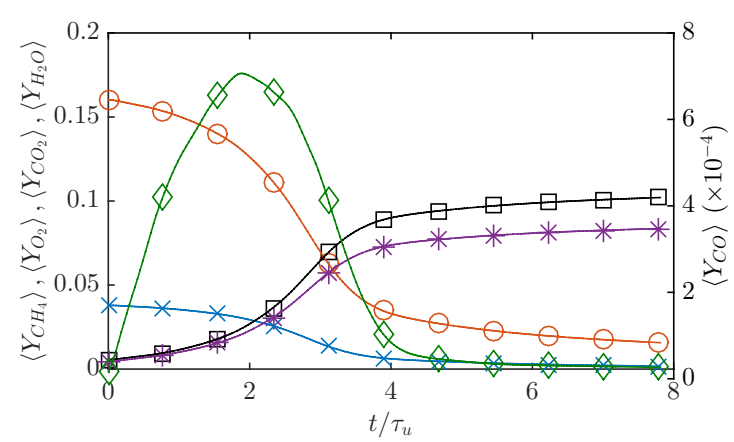

(c)

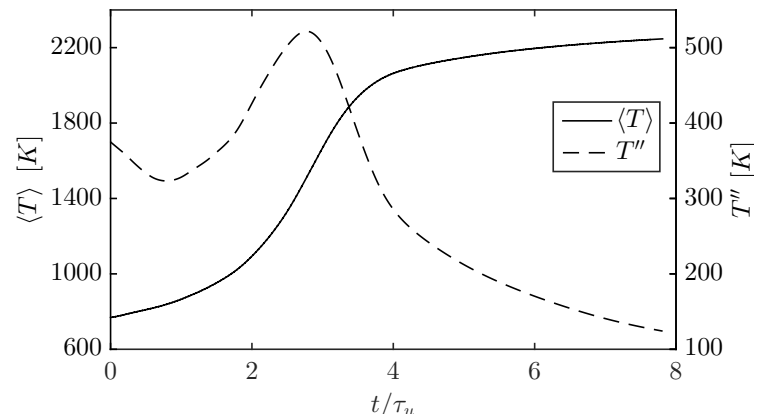

(b)

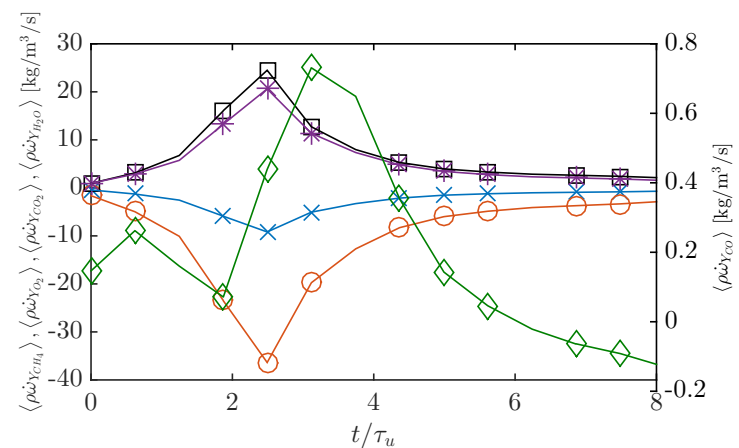

(d)

Figure 7: (Color online) Temporal evolution of (a) turbulent kinetic energy and dissipation rate; (b) mean and RMS of temperature; (c) species mass fractions; and (d) mean chemical source terms. The legends in panel (c) and (d) follow: $\mathrm{CH}_{4}$ $(\times), \mathrm{O}_{2}(\bigcirc), \mathrm{CO}(\diamond), \mathrm{CO}_{2}(\square), \mathrm{H}_{2} \mathrm{O}(*)$.

Figure 8 shows the flame index [34] of the flow field, which is computed as the scalar product between the gradient of the fuel and oxidizer mass fraction:

$$
G_{\mathrm{FO}}=\frac{\nabla Y_{\mathrm{CH}_{4}} \cdot \nabla Y_{\mathrm{O}_{2}}}{\left|\nabla Y_{\mathrm{CH}_{4}}\right| \cdot\left|\nabla Y_{\mathrm{O}_{2}}\right|} .
$$




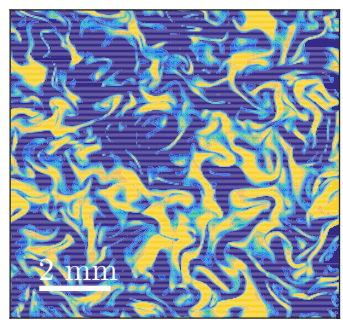

(a) $t / \tau_{u}=0.4$

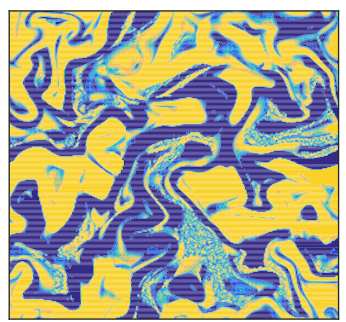

(b) $t / \tau_{u}=2.9$

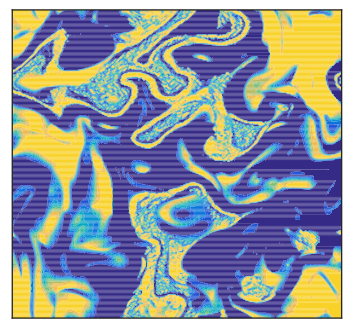

(c) $t / \tau_{u}=4.8$

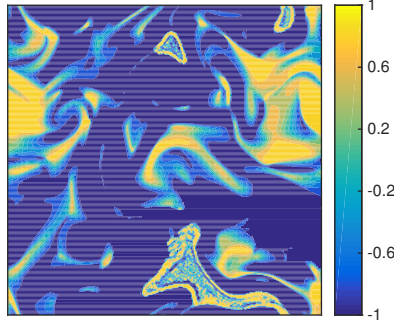

(d) $t / \tau_{u}=7.9$

Figure 8: (Color online) Flame index $G_{\mathrm{FO}}$ at $t / \tau_{u}=\{0.4,2.9,4.8,7.9\}$.

When $G_{\mathrm{FO}}=1$, the gradient of the fuel and oxidizer mass fraction is perfectly aligned, indicating a premixed flame; on the contrary, when $G_{\mathrm{FO}}=-1$, the gradient of the fuel and oxidizer mass fraction is in the opposite direction, indicating a diffusion flame. In Fig. 8(a), we can see that the flame consists of separate regions of premixed and diffusion flames at the initial stage of the combustion process. The region with premixed flame starts to grow with time and becomes connected. The flow field with maximum premixed regions is observed at around three eddy-turnover times, as shown in Fig. 8(b), which correlates with the occurrence of the maximum reaction rate. A decrease in proportion of the premixed flame region is observed after three eddy-turnover times, as shown in Figs. 8(c) and 8(d). The flow field hence becomes diffusion-dominant.

The DNS results show a complex flow structure. The flame topology is represented by a combination of premixed and diffusion combustion modes, and the relative proportion of this mixture changes with time. In addition, the flame structure is disrupted by the turbulent flow field. This makes the case particularly suitable for testing the robustness of the proposed turbulence closure model.

\section{A priori analysis of deconvolution methods}

In this a priori study, we consider the results corresponding to the instantaneous flow field at $t / \tau_{u}=2.9$, as shown in Fig. 8(b). This flow field consists of a complex flow structure, where premixed and nonpremixed flame regions coexist and the turbulence intensity is strong. In the following analysis, we use a three-dimensional top-hat filter to filter the scalars. The performance of ADM and RDM is investigated over a range of filter widths, $\Delta / \delta_{s}$, through direct comparison with filtered DNS solutions. The performance of these different methods is evaluated by assessing their accuracy in reconstructing scalars, and in closing turbulent-scalar fluxes and filtered chemical source terms. As a reference, terms computed using ADM and RDM are compared to those that are directly evaluated using Favre-filtered scalars, which we denoted as "no model".

\subsection{Deconvolution and reconstruction of scalars}

Because the chemical reaction in turbulent combustion is localized at small scales, it is critical to accurately represent reactive scalars at high wavenumbers. We therefore conduct direct assessments of the deconvolved scalars in terms of physical structure and spectral content.

\subsubsection{Scalar reconstruction error}

Figure 9 shows a comparison of reconstructed flow field results we obtained from explicitly filtered DNS solutions with filter widths of $\Delta / \delta_{s}=\{0.2,1.0,5.0\}$. The "DNS" column in Fig. 9 shows the unfiltered $\mathrm{CO}$ mass fraction field, where a localized flame structure is observed. The "Filtered" column shows the filtered CO mass fraction corresponding to three test filter widths. Our results show that the small-scale structures are filtered out with increasing filter width. The "ADM" and "RDM" columns of this figure show the CO mass fraction reconstructed for each method, respectively. When $\Delta \leq \delta_{s}$, both methods provide comparable results. However, with increased filter width, the reconstructed flame structure using ADM 


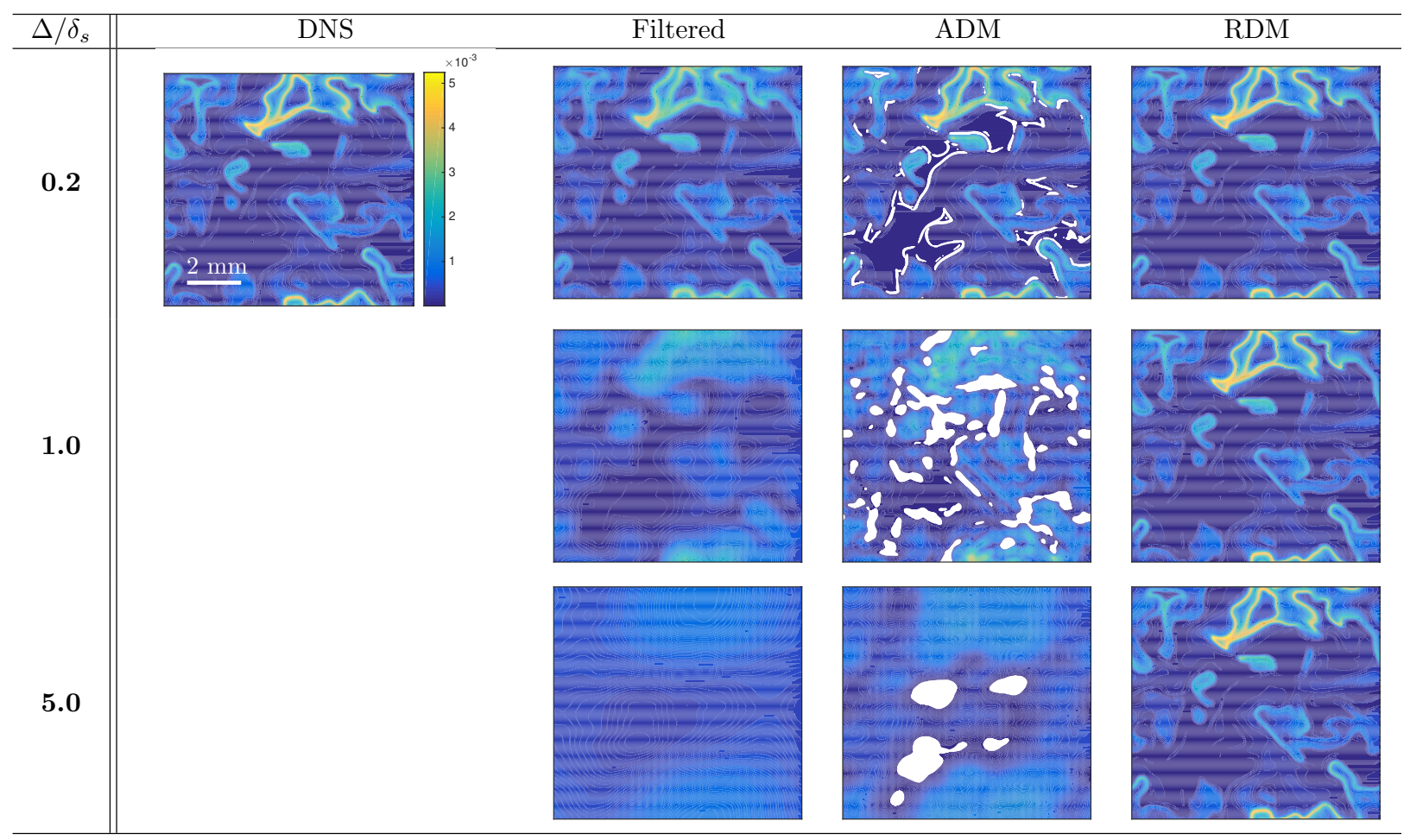

Figure 9: (Color online) Reconstructed CO mass fraction using ADM and RDM for different filter widths at $t / \tau_{u}=2.9$. The blanked-out regions correspond to negative $\mathrm{CO}$ mass fraction.

introduces spurious oscillations. In proximity to the flame, negative CO mass fractions are generated, as represented by the blanked-out regions in the "ADM" column. In contrast, we observe excellent agreement in the flame structure using RDM for all three filter widths.

To assess the accuracy of ADM and RDM, we quantitatively compare the normalized scalar reconstruction error, which is defined as:

$$
\epsilon(\phi)=\frac{\left\|\phi-\phi^{\star}\right\|_{2}}{\|\phi\|_{2}}
$$

A comparison of the error between reconstructed flow-field quantities and filtered DNS solutions for velocity, species mass fractions and temperature is presented in Fig. 10 for three different filter widths. In general, we observe an increase in the reconstruction error as the filter width increases for all quantities. Additionally, the error associated with RDM is substantially lower than with ADM for all filter widths. One point to note for the reconstruction error when using either "no model" or ADM is that the error in the CO mass fraction is largest at $\Delta / \delta_{s}=0.2$, as shown in Fig. 10(a). With increasing filter width, the difference in the reconstruction error among scalars diminishes, as shown in Figs. 10(b) and 10(c). This results from the underlying structure of the scalars, and their relationship with the filter width. In Fourier space, the deconvolution operation amplifies the filtered signal such that the damping at each wavenumber caused by the low-pass filter is canceled. Because of the nature of the low-pass filter, the higher the wavenumber, the more damping is applied to the signal; therefore a larger amplification factor is required in the deconvolution operation to reconstruct the signal. Consequently, the deconvolution operation is more sensitive to errors at high wavenumbers. Signals with more localized structures in physical space are less smooth and have more energy at high wavenumbers; therefore they are more sensitive to the filter and deconvolution operations. As the filter width increases, the cutoff wavenumber decreases; therefore the length scale that will be influenced by the deconvolution operation increases. In terms of reactive scalars, minor species have localized structures whereas the major species have broader distributions. Because of this, the reconstruction error 


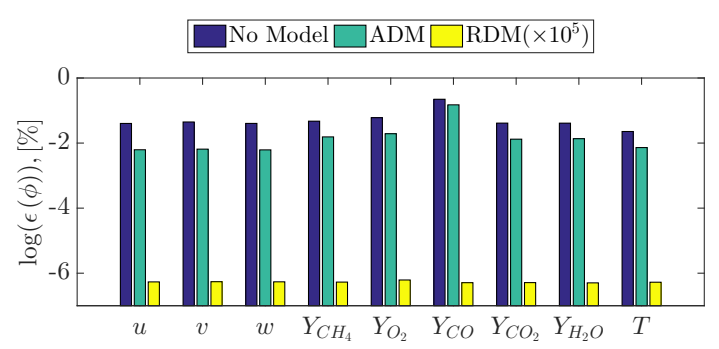

(a) $\Delta / \delta_{s}=0.2$

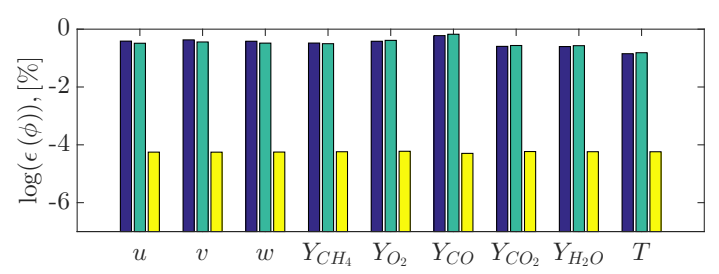

(b) $\Delta / \delta_{s}=1.0$

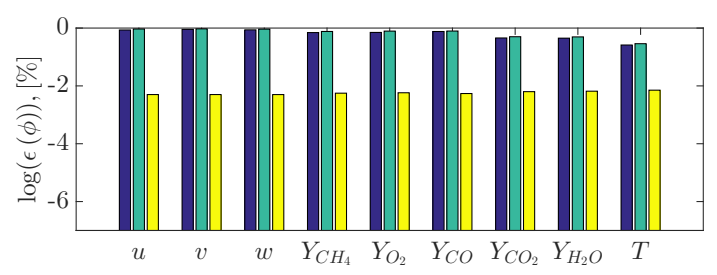

(c) $\Delta / \delta_{s}=5.0$

Figure 10: (Color online) Comparison of normalized error for deconvolved velocity, reactive scalars and temperature at $t / \tau_{u}=$ 2.9 using "no model", ADM and RDM with $\Delta / \delta_{s}=\{0.2,1.0,5.0\}$. Note that the error generated by RDM is multiplied for $10^{5}$ for graphical presentation.

for $\Delta / \delta_{s}=0.2$ is larger for the $\mathrm{CO}$ mass fraction than for other scalars. For conditions where $\Delta$ is comparable to the structure of the major species, the deconvolution error becomes significant. On the other hand, we do not observe this phenomenon when RDM is used. This indicates that RDM improves the accuracy of reconstructing signals above the cutoff wavenumber. This also indicates that RDM is insensitive to the smoothness and locality of the scalars' structure. These properties are particularly useful for the reconstruction of minor-species profiles.

Note that the reconstruction error in the CO mass fraction is more sensitive to the change in filter width at small scales, whereas reconstructed major species and temperature behave similarly for each deconvolution method. Therefore, only results for the CO mass fraction and temperature are discussed in the following analysis.

To further illustrate the relationship between reconstruction error and filter width, we plot the reconstruction error for the CO mass fraction and temperature as a function of $\Delta / \delta_{s}$ for ADM and RDM in Fig. 11. As a general trend for all models, the scalar reconstruction error increases with filter width. For the case without reconstruction ("no model"), the error reaches its asymptotic limit at $\Delta / \delta_{s}=1$ and becomes of order unity. As the filter width increases, the error for the reconstructed scalars using ADM increases monotonically towards the asymptotic error for the case without model. The results obtained for $\Delta / \delta_{s}<1$ show a faster increase in the error with RDM than with ADM. Asymptotic limits of the error are reached at $\Delta / \delta_{s}=2$ for all deconvolution methods. We observe that the error of RDM is more than seven orders of magnitude smaller than the error without model. This result demonstrates the capability of RDM in reconstructing filtered scalars above the cutoff wavenumber. 


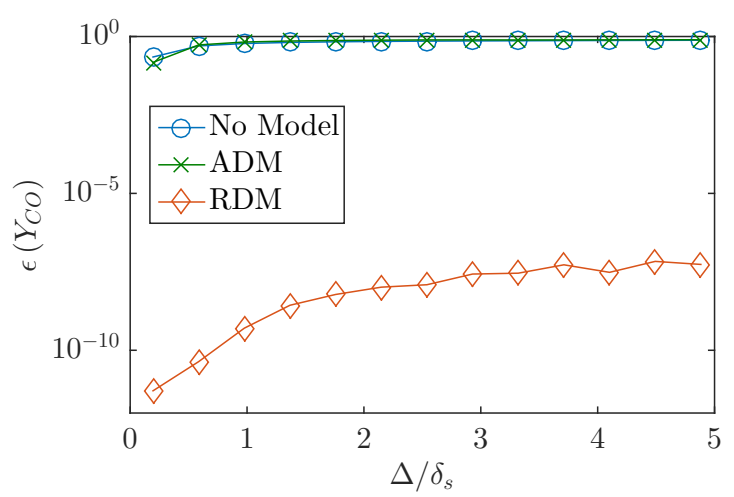

(a) CO mass fraction

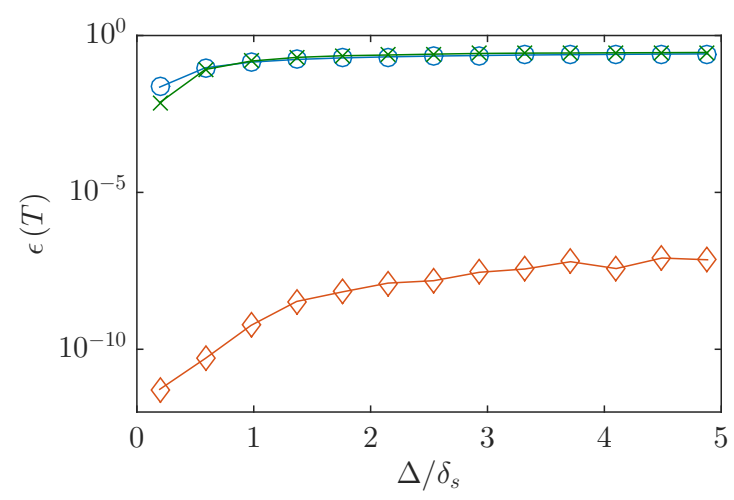

(b) Temperature

Figure 11: (Color online) Normalized reconstruction errors for (a) CO mass fraction and (b) temperature obtained with "no model", ADM and RDM.

Figure 12 shows distributions of the reconstructed CO mass fraction and temperature, which are normalized by the DNS solutions. The corresponding statistics are shown in Table 1. For both scalars, the variance of the distribution increases with filter width for cases without model and with ADM. The distribution associated with RDM remains close to a $\delta$-function in all cases. Specifically, considering the distribution of point-wise DNS normalized temperature $T^{\widetilde{ }} / T$ for the case where $\Delta / \delta_{s}=0.2$, we observe that the distribution for "no model" is close to a Laplace distribution with a mean value of $\left\langle T^{\widetilde{\star}} / T\right\rangle=9.9723 \times 10^{-1}$ and variance $6.0134 \times 10^{-4}$. With $\mathrm{ADM}$, the mean and variance for $T^{\widetilde{\star}} / T$ is $9.9994 \times 10^{-1}$ and $4.7903 \times 10^{-5}$. For RDM, the distribution of $T^{\widetilde{\star}} / T$ is a $\delta$-function centered at unity. As $\Delta / \delta_{s}$ increases, the variance of the distribution of $T^{\widetilde{\star}} / T$ increases for cases without model as well as with ADM, indicating that the deconvolved temperature starts to deviate from DNS. The smaller variance observed for cases with ADM compared to cases without model shows an improvement in matching the DNS with ADM. Further increasing $\Delta / \delta_{s}$ to 5.0 leads to an under-prediction of the temperature for cases without model and with ADM. This illustrates that $\mathrm{ADM}$ is not efficient in reconstructing the temperature when the filter width is large compared to the characteristic length scale. In contrast, the change in the distribution of $T^{\widetilde{\star}} / T$ with respect to the filter width is not observed for cases employing RDM.

\begin{tabular}{|c|c|c|c|c|c|}
\hline & & & No Model & ADM & RDM \\
\hline \multirow{4}{*}{$\Delta / \delta_{s}=0.2$} & \multirow{2}{*}{$Y_{\mathrm{CO}}^{\widetilde{\star}} / Y_{\mathrm{CO}}$} & mean & $1.1909 \times 10^{0}$ & $1.0122 \times 10^{0}$ & $1.0000 \times 10^{0}$ \\
\hline & & variance & $2.7760 \times 10^{-1}$ & $2.1731 \times 10^{-1}$ & $0.0000 \times 10^{0}$ \\
\hline & \multirow{2}{*}{$T^{\widetilde{\star}} / T$} & mean & $9.9723 \times 10^{-1}$ & $9.9994 \times 10^{-1}$ & $1.0000 \times 10^{0}$ \\
\hline & & variance & $6.0134 \times 10^{-4}$ & $4.7903 \times 10^{-5}$ & $0.0000 \times 10^{0}$ \\
\hline \multirow{4}{*}{$\Delta / \delta_{s}=1.0$} & \multirow{2}{*}{$Y_{\mathrm{CO}}^{\widetilde{\star}} / Y_{\mathrm{CO}}$} & mean & $1.3433 \times 10^{0}$ & $1.0948 \times 10^{0}$ & $1.0000 \times 10^{0}$ \\
\hline & & variance & $6.7943 \times 10^{-1}$ & $9.1662 \times 10^{-1}$ & $0.0000 \times 10^{0}$ \\
\hline & \multirow{2}{*}{$T^{\widetilde{\star}} / T$} & mean & $9.8791 \times 10^{-1}$ & $1.0064 \times 10^{0}$ & $1.0000 \times 10^{0}$ \\
\hline & & variance & $2.5741 \times 10^{-2}$ & $2.5723 \times 10^{-2}$ & $0.0000 \times 10^{0}$ \\
\hline \multirow{4}{*}{$\Delta / \delta_{s}=5.0$} & \multirow{2}{*}{$Y_{\mathrm{CO}}^{\widetilde{\star}} / Y_{\mathrm{CO}}$} & mean & $1.4107 \times 10^{0}$ & $1.2327 \times 10^{0}$ & $1.0000 \times 10^{0}$ \\
\hline & & variance & $1.0518 \times 10^{0}$ & $1.0742 \times 10^{0}$ & $0.0000 \times 10^{0}$ \\
\hline & \multirow{2}{*}{$T^{\widetilde{\star}} / T$} & mean & $9.8821 \times 10^{-1}$ & $9.9913 \times 10^{-1}$ & $1.0000 \times 10^{0}$ \\
\hline & & variance & $1.0182 \times 10^{-1}$ & $1.0634 \times 10^{-1}$ & $0.0000 \times 10^{0}$ \\
\hline
\end{tabular}

Table 1: Statistics for $Y_{\mathrm{CO}}^{\widetilde{x}} / Y_{\mathrm{CO}}$ and $T^{\widetilde{x}} / T$ at $\Delta / \delta_{s}=\{0.2,1.0,5.0\}$ using "no model", ADM and RDM.

Note that the distribution of $Y_{\mathrm{CO}}^{\widetilde{\star}} / Y_{\mathrm{CO}}$ has a larger variance compared to the distribution of $T^{\widetilde{\star}} / T$ without model and with ADM. This indicates that the error in the reconstructed CO mass fraction is larger 


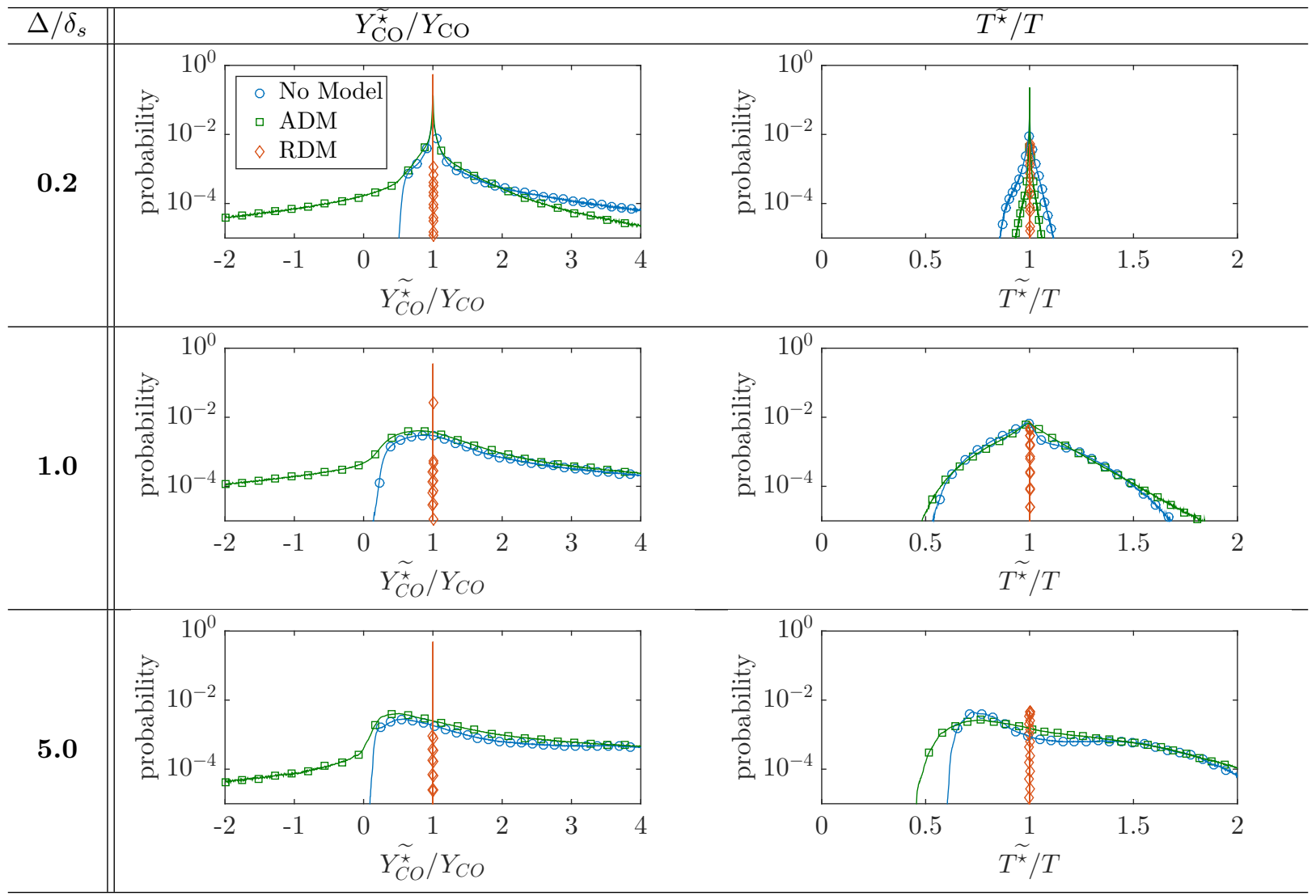

Figure 12: (Color online) Comparison of probability density function of normalized deconvolved scalars for (left) $Y_{\mathrm{CO}}^{\widetilde{\star}} / Y_{\mathrm{CO}}$ and (right) $T^{\widetilde{ }} / T$ for three different values of $\Delta / \delta_{s}$.

than that of $T$, which is consistent with observations from Fig. 10. For the case with filter width $\Delta / \delta_{s}=0.2$, the normalized scalar obtained from both ADM and "no model" has a distribution with unity mean. The variance of the distribution with $\mathrm{ADM}$ is three times lower than the variance for the case without model. With increasing filter width, the mean value of the distribution for $Y_{\mathrm{CO}}^{\widetilde{\star}} / Y_{\mathrm{CO}}$ shifts towards zero and the variance grows for cases without model. This is because of the effective diffusion caused by the filtering operation. With $\mathrm{ADM}$, the mean of the distribution for $Y_{\mathrm{CO}}^{\widetilde{\star}} / Y_{\mathrm{CO}}$ tilts towards unity and the variance is reduced. However, we observe a sign reversal at the left tail of the distribution associated with ADM, which indicates the existence of negative species mass fractions. In comparison, the results show stable performance with RDM for different filter widths where we see a distribution close to a $\delta$-function in all cases.

\subsubsection{Spectral content}

We further examine the effectiveness of applying deconvolution methods by the amount of signal that is recovered at high wavenumbers. For this, we evaluate the turbulent kinetic energy spectrum using different methods for cases with filter width $\Delta / \delta_{s}=\{0.2,1.0,5.0\}$, and the results are shown in Fig. 13. As the filter width increases, the cutoff wavenumber is reduced. For cases with filter width $\Delta / \delta_{s}=0.2$ and 1.0 , both $\mathrm{ADM}$ and RDM provide good agreement with DNS. However, for the filter width $\Delta / \delta_{s}=5.0$, only RDM fully recovers the energy spectrum.

The difference in recovered signal between $\mathrm{ADM}$ and $\mathrm{RDM}$ is more substantial for reactive scalars. Figure 14 compares the energy spectrum of the reconstructed CO mass fraction using different methods for cases with filter width $\Delta / \delta_{s}=\{0.2,1.0,5.0\}$. The filtered CO mass fraction without deconvolution 


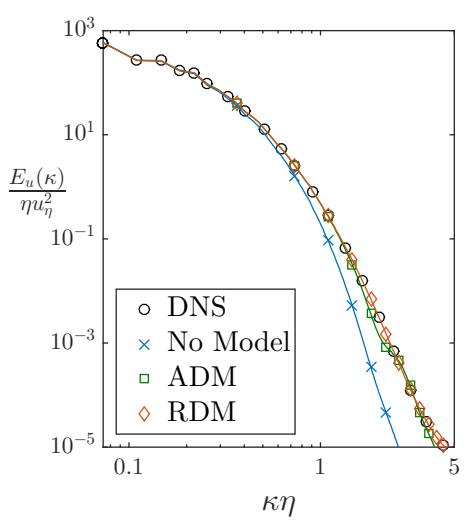

(a) $\Delta / \delta_{s}=0.2$

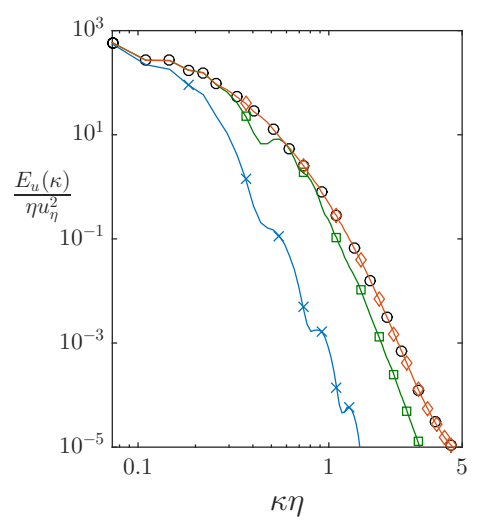

(b) $\Delta / \delta_{s}=1.0$

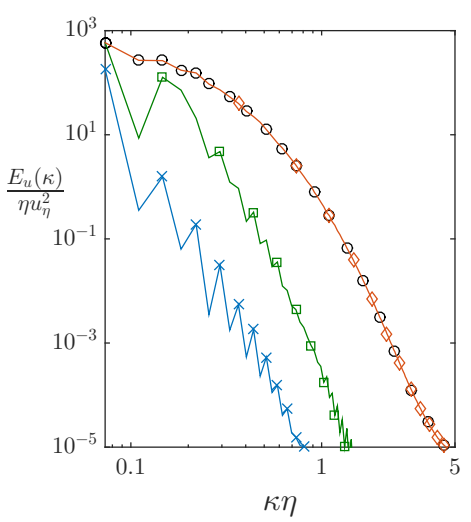

(c) $\Delta / \delta_{s}=5.0$

Figure 13: (Color online) Spectrum of turbulent kinetic energy for DNS, "no model", ADM, and RDM.

shows a higher level of energy loss as the filter width increases. This is similar to what we observe for the turbulent kinetic energy. With ADM, the energy spectrum is restored for the case $\Delta / \delta_{s}=0.2$, with a small discrepancy at high wavenumbers. The amount of unrecovered signal grows with increasing filter width. This result explains our observation that, when using ADM, the error in scalar reconstruction increases as the filter width increases. In contrast, we observe energy spectra from RDM that are consistent with the DNS results for all filter widths.

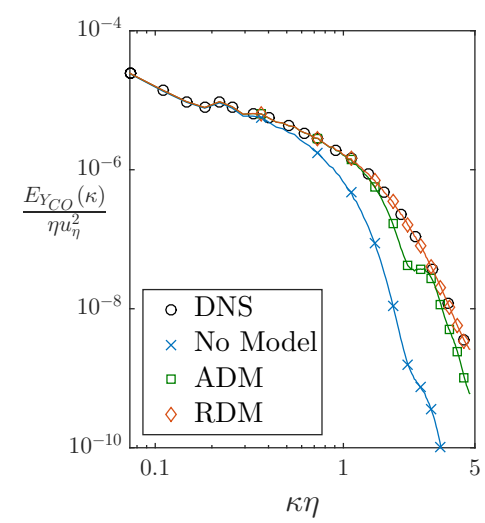

(a) $\Delta / \delta_{s}=0.2$

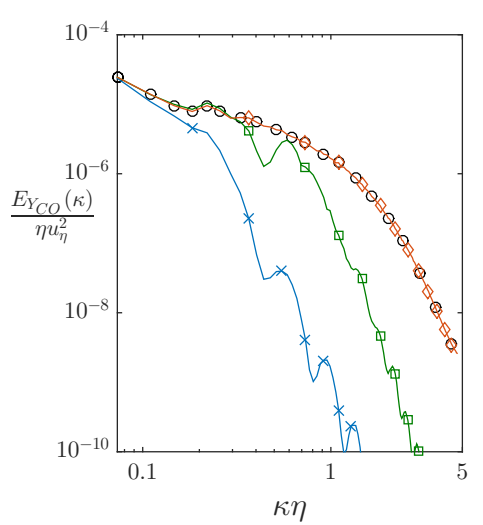

(b) $\Delta / \delta_{s}=1.0$

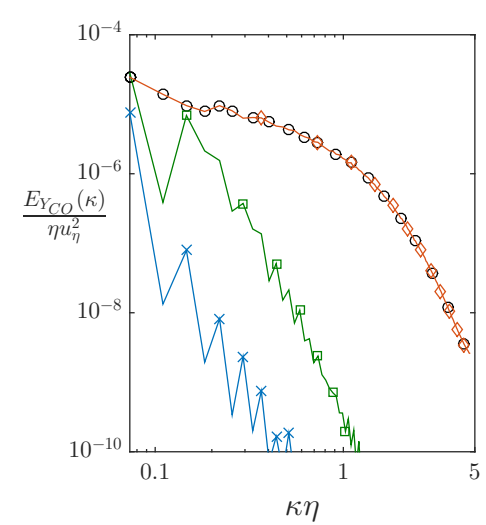

(c) $\Delta / \delta_{s}=5.0$

Figure 14: (Color online) Spectrum of CO mass fraction for DNS, "no model", ADM, and RDM.

Results for scalar reconstruction using RDM show that the method is stable for all filter widths. The fine-scale flame structure is recovered by RDM irrespective of the locality and smoothness of the scalar field.

\subsection{Turbulent scalar mixing term in LES equation}

We proceed by analyzing the turbulent scalar mixing term using deconvolution methods computed from Eq. (11b). Figure 15 compares $\sigma_{Y_{\mathrm{CO}}}$ computed using ADM and RDM to $\sigma_{Y_{\mathrm{CO}}}$ computed using DNS solutions with filter width $\Delta / \delta_{s}=\{0.2,1.0,5.0\}$. From these results, we see that the magnitude of $\sigma_{Y_{\mathrm{CO}}}$ is underpredicted when a deconvolution model is not applied. In addition, the structure of $\sigma_{Y_{\mathrm{CO}}}$ is mispredicted starting at $\Delta / \delta_{s}=1.0$. With $\mathrm{ADM}$, the magnitude of $\sigma_{Y_{\mathrm{CO}}}$ is predicted correctly. However, the structure 


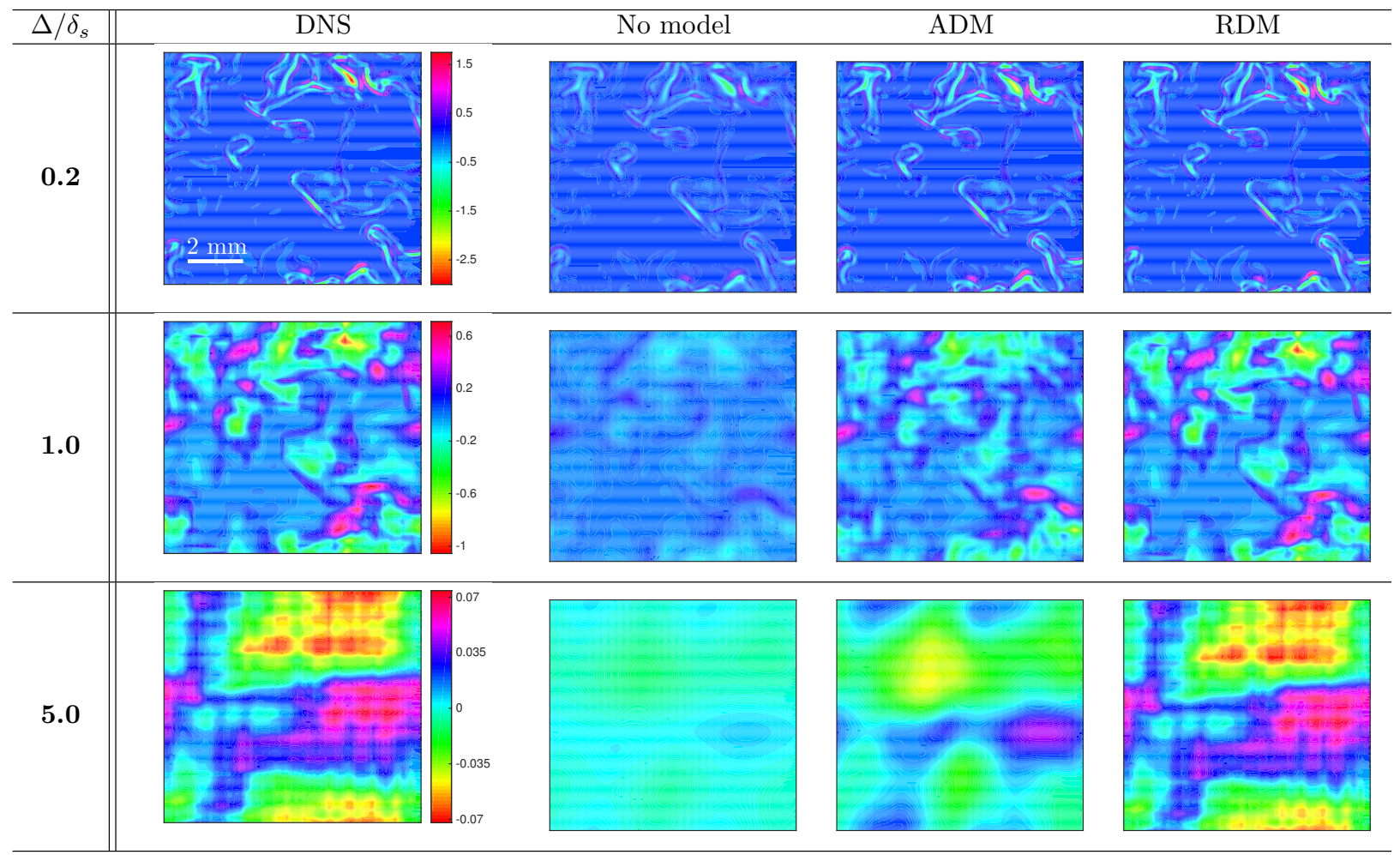

Figure 15: (Color online) Turbulent scalar mixing term $\sigma_{Y_{\mathrm{CO}}}$ evaluated from Eq. (11b) with different filter widths computed using "no model", ADM and RDM.

of $\sigma_{Y_{\mathrm{CO}}}$ remains similar to cases without a deconvolution model. With RDM, both the magnitude and structure of $\sigma_{Y_{\mathrm{CO}}}$ matches the DNS results.

Figure 16 shows a quantitative comparisons of $\sigma_{Y_{\mathrm{CO}}}$ and $\sigma_{T}$ obtained from "no model", ADM and RDM to DNS. The statistical results of $\sigma_{\phi \text {,model }}$ that are normalized by the corresponding DNS data are shown in Table 2. For cases without model, the magnitude of $\sigma_{\phi}$ is under-predicted. This is a direct consequence of the reduction in gradient caused by filtering. With $\mathrm{ADM}, \sigma_{\phi}$ is predicted correctly for $\Delta / \delta_{s}=0.2$. The variance of $\sigma_{\phi, \mathrm{ADM}} / \sigma_{\phi, \mathrm{DNS}}$ increases for cases when $\Delta / \delta_{s}=1.0$ and 5.0, which results from the misprediction in the structure of $\sigma_{\phi}$ when using $\mathrm{ADM}$, as shown in Fig. 15 . In contrast, when using RDM, $\sigma_{\phi, \text { RDM }}$ matches $\sigma_{\phi, \mathrm{DNS}}$ for all filter widths with a distribution of $\sigma_{\phi, \mathrm{RDM}} / \sigma_{\phi, \mathrm{DNS}}$ close to a $\delta$-function for all cases.

\subsection{Reconstruction of filtered chemical source term}

The filtered chemical source term $\overline{\rho \dot{\omega}_{\phi}(\boldsymbol{Y}, T)}$ in Eq. (7c) is a function of mass fractions of the reactive scalars and temperature. With the deconvolution model, scalars are reconstructed from filtered quantities that are solved in the LES equations. Therefore, the closure of the turbulent chemical source term using deconvolution methods can be computed as $\overline{\rho \dot{\omega}_{\phi}\left(\boldsymbol{Y}^{\widetilde{*}}, T^{\widetilde{\star}}\right)}$.

Figure 17 shows the filtered chemical source term of $\mathrm{CO}$ as a function of the filter width $\Delta / \delta_{s}$. We see from the filtered DNS solutions in the "DNS" column that the flame structure is filtered out as $\Delta / \delta_{s}$ increases. The "No model" column shows the corresponding results without model. We observe an overprediction in source term magnitude and a misprediction in flame topology in these cases. The reason is that the filtering causes an equivalent effect of diffusion of the scalars. From the third column, it is found that ADM reduces the error in the chemical source term by a factor of two, which is because the locality of the scalars are weakly recovered. However, because the reconstruction of the scalar structure does not match the DNS solution, the flame topology obtained using ADM is different from the original. In contrast, 


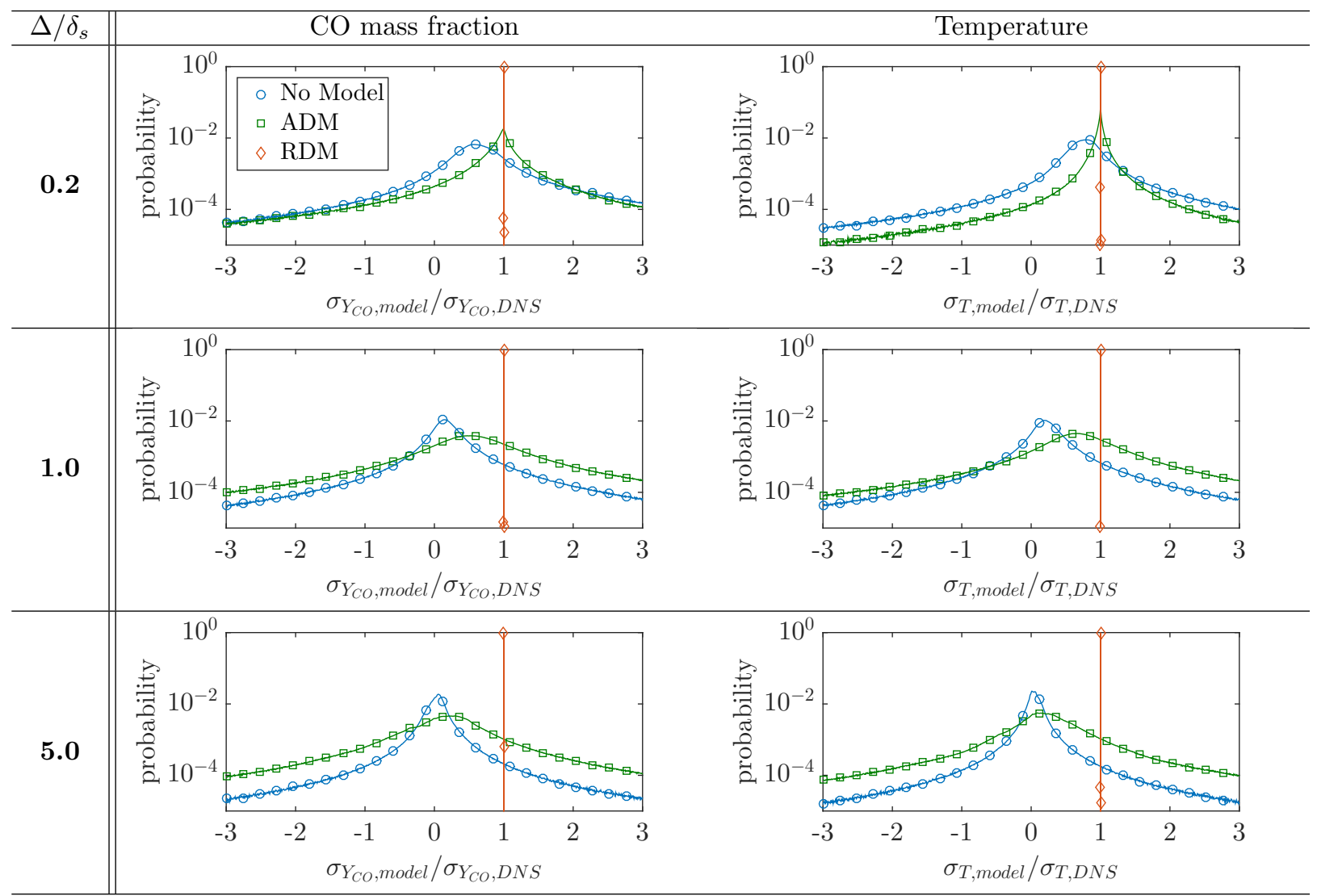

Figure 16: (Color online) Comparison of probability density function of normalized turbulent scalar mixing term for (left) CO mass fraction and (right) temperature as a function of $\Delta / \delta_{s}$.

\begin{tabular}{|c|c|c|c|c|c|}
\hline & & & No Model & ADM & RDM \\
\hline \multirow{3}{*}{$\Delta / \delta_{s}=0.2$} & $\mathrm{CO}$ mass fraction & mean & $6.2293 \times 10^{-1}$ & $9.0112 \times 10^{-1}$ & $1.0000 \times 10^{0}$ \\
\hline & & variance & $5.1401 \times 10^{-1}$ & $4.2451 \times 10^{-1}$ & $0.0000 \times 10^{0}$ \\
\hline & Temperature & mean & $7.5563 \times 10^{-1}$ & $9.8374 \times 10^{-1}$ & $1.0000 \times 10^{0}$ \\
\hline \multirow{3}{*}{$\Delta / \delta_{s}=1.0$} & & & $1.8524 \times 10^{-1}$ & $4.7272 \times 10^{-1}$ & $1.0000 \times 10^{0}$ \\
\hline & $\mathrm{CO}$ mass fraction & variance & $3.8433 \times 10^{-1}$ & $9.1932 \times 10^{-1}$ & $0.0000 \times 10^{0}$ \\
\hline & Temperature & $\begin{array}{c}\text { mean } \\
\text { variance }\end{array}$ & $\begin{array}{l}2.2151 \times 10^{-1} \\
3.8871 \times 10^{-1}\end{array}$ & $\begin{array}{l}6.1663 \times 10^{-1} \\
8.2204 \times 10^{-1}\end{array}$ & $\begin{array}{l}1.0004 \times 10^{0} \\
0.0000 \times 10^{0}\end{array}$ \\
\hline \multirow{2}{*}{$\Delta / \delta_{s}=5.0$} & $\mathrm{CO}$ mass fraction & $\begin{array}{c}\text { mean } \\
\text { variance }\end{array}$ & $\begin{array}{l}3.3804 \times 10^{-2} \\
1.8504 \times 10^{-1}\end{array}$ & $\begin{array}{l}1.4950 \times 10^{-1} \\
7.4403 \times 10^{-1}\end{array}$ & $\begin{array}{c}9.9993 \times 10^{-1} \\
0.0000 \times 10^{0}\end{array}$ \\
\hline & Temperature & $\begin{array}{c}\text { mean } \\
\text { variance }\end{array}$ & $\begin{array}{l}4.9132 \times 10^{-2} \\
1.4990 \times 10^{-1}\end{array}$ & $\begin{array}{l}1.9402 \times 10^{-1} \\
6.2431 \times 10^{-1}\end{array}$ & $\begin{array}{l}1.0006 \times 10^{0} \\
0.0000 \times 10^{0}\end{array}$ \\
\hline
\end{tabular}

Table 2: Statistics for $\sigma_{Y_{\mathrm{CO} \text {, model }}} / \sigma_{Y_{\mathrm{CO}, \mathrm{DNS}}}$ and $\sigma_{T, \text { model }} / \sigma_{T, \mathrm{DNS}}$ at $\Delta / \delta_{s}=\{0.2,1.0,5.0\}$ using "no model", ADM and RDM.

the chemical source term computed using RDM matches the DNS results qualitatively in terms of both topology and magnitude, as shown in the last column of Fig. 17.

A comparison of the distribution of the chemical source term of the CO mass fraction is shown in Fig. 18. The chemical source term is computed using different deconvolution closures normalized locally by 


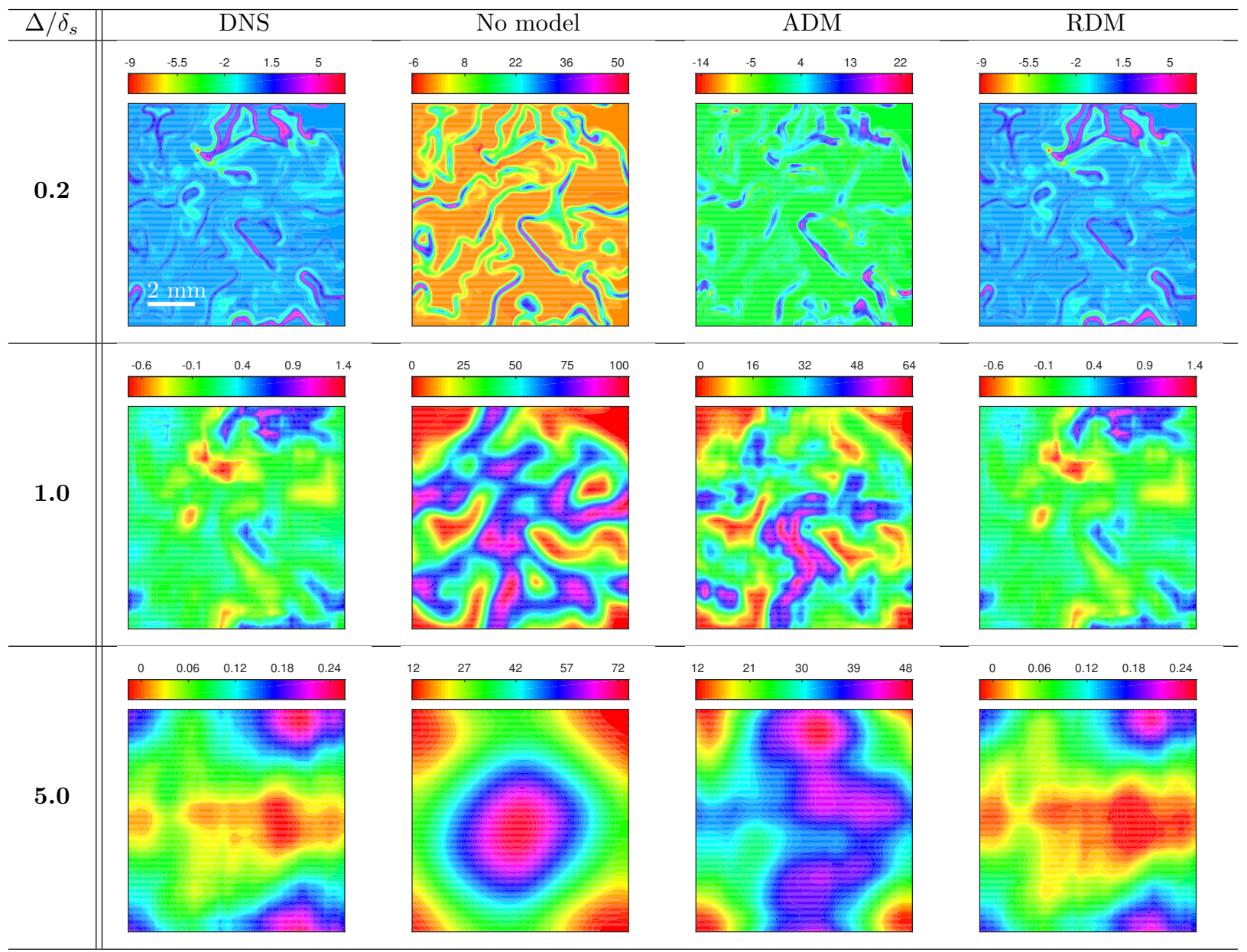

Figure 17: (Color online) Chemical source term $\bar{\rho}_{Y_{\mathrm{CO}}}$ for different filter widths computed using "no model", ADM and RDM, with units $\left[\mathrm{kg} /\left(\mathrm{m}^{3} \mathrm{~s}\right)\right]$.

the filtered DNS solution. The statistics of $\overline{\rho \dot{\omega}_{Y_{\mathrm{CO}}}\left(\phi^{\widetilde{*}}\right)} / \overline{\rho \dot{\omega}_{Y_{\mathrm{CO}}}(\phi)}$ are shown in Table 3. In Fig. 18, the peak of the distributions for all methods are centered at unity for the case with $\Delta / \delta_{s}=0.2$. The distribution for $\overline{\rho \dot{\omega}_{Y_{\mathrm{CO}}}\left(\phi^{\widetilde{*}}\right)} / \overline{\rho \dot{\omega}_{Y_{\mathrm{CO}}}(\phi)}$ spreads for cases without model and with ADM as the filter width increases, with the mean shifting away from unity. In particular, for the filter width of $\Delta / \delta_{s}=1.0$ the mean and variance are 43.0 and 42.7 for no model, and 40.4 and 37.4 for ADM, respectively. These values increase to 354.9 and 2638.1 for no model, and 324.1 and 2159.2 for $\mathrm{ADM}$ for $\Delta / \delta_{s}=5.0$. In addition, the results for cases without model and with ADM show incorrect predictions for the consumption and production rates. With RDM, the mean of the distribution of $\overline{\rho \dot{\omega}_{Y_{\mathrm{CO}}}\left(\phi^{\widetilde{*}}\right)} / \overline{\rho \dot{\omega}_{Y_{\mathrm{CO}}}(\phi)}$ is unity for all filter widths, while the variance remains below unity.

In summary, the results show that RDM is accurate in predicting both turbulent mixing and filtered chemical source terms based on our a priori analysis. This illustrates the potential of using RDM as a closure model in combustion LES.

\section{A posteriori analysis of $\mathrm{RDM}$}

To analyze the temporal stability and accuracy of RDM, we complement the a priori analysis by conducting an a posteriori study of the scalars on the configurations discussed in Sec. 4. In this study, we perform 


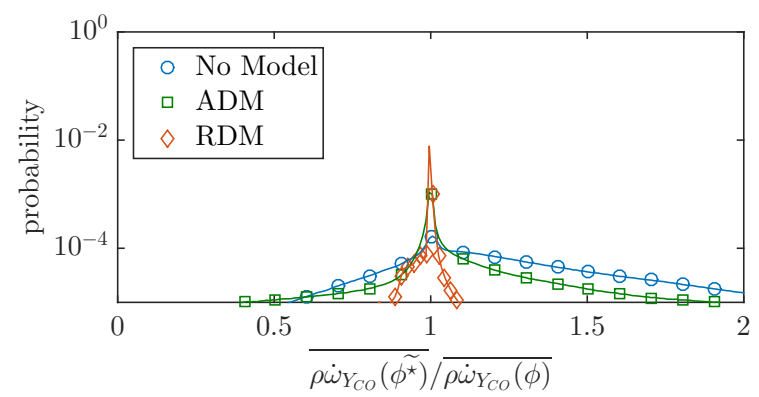

(a) $\Delta / \delta_{s}=0.2$

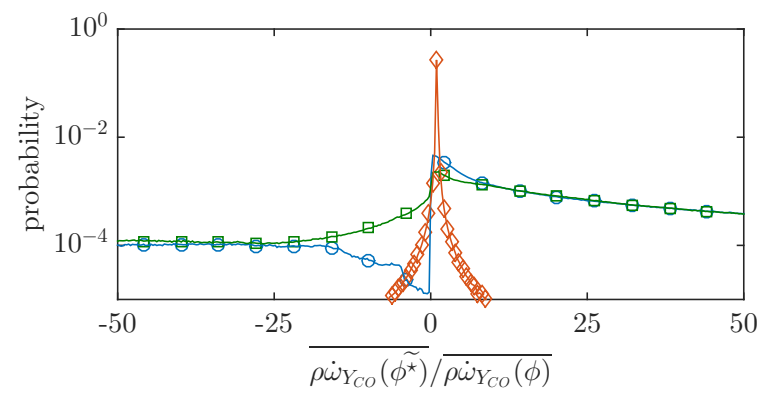

(b) $\Delta / \delta_{s}=1.0$

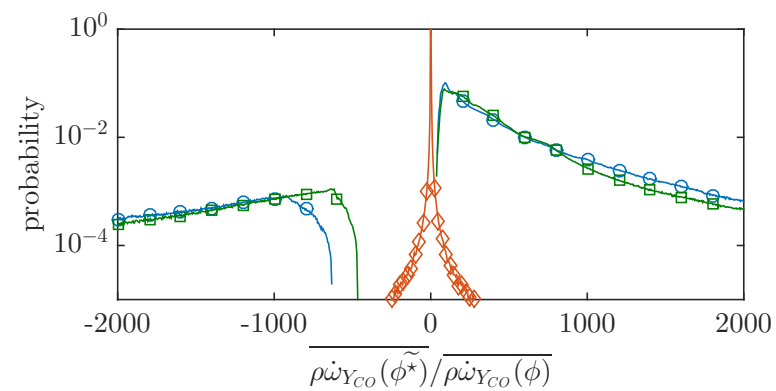

(c) $\Delta / \delta_{s}=5.0$

Figure 18: (Color online) Comparison of turbulent chemical source term for $\mathrm{CO}$ mass fraction between DNS and reconstruction using "no model", ADM and RDM.

\begin{tabular}{|c|c|c|c|c|}
\cline { 3 - 5 } \multicolumn{2}{c|}{} & No Model & ADM & RDM \\
\hline \multirow{2}{*}{$\Delta / \delta_{s}=0.2$} & mean & $8.6512 \times 10^{-1}$ & $1.0005 \times 10^{0}$ & $9.9334 \times 10^{-1}$ \\
& variance & $2.8415 \times 10^{0}$ & $2.3485 \times 10^{0}$ & $1.2503 \times 10^{-2}$ \\
\hline \multirow{2}{*}{$\Delta / \delta_{s}=1.0$} & mean & $4.2993 \times 10^{1}$ & $4.0368 \times 10^{1}$ & $9.4481 \times 10^{-1}$ \\
& variance & $4.2698 \times 10^{1}$ & $3.7356 \times 10^{1}$ & $2.0930 \times 10^{-1}$ \\
\hline \multirow{2}{*}{$\Delta / \delta_{s}=5.0$} & mean & $3.5493 \times 10^{2}$ & $3.2412 \times 10^{2}$ & $8.7161 \times 10^{-1}$ \\
& variance & $2.6381 \times 10^{2}$ & $2.1592 \times 10^{2}$ & $3.4670 \times 10^{-1}$ \\
\hline
\end{tabular}

Table 3: Statistics for $\overline{\rho \dot{\omega}_{Y_{\mathrm{CO}}}\left(\phi^{\widetilde{*}}\right)} / \overline{\rho \dot{\omega}_{Y_{\mathrm{CO}}}(\phi)}$ for $\Delta / \delta_{s}=\{0.2,1.0,5.0\}$ using "no model", ADM and RDM.

simulations on a $256^{3}$ grid. Equations (12) are solved with a low-Mach variable-density formulation. In our current approach, we solve the scalar transport equations with explicit filtering and applied deconvolution methods to the scalars. The momentum equations are solved in the context of implicit filtering and we take no closure model for the Reynolds stress gradient term by assuming $u_{i}^{\star}=\widetilde{u}_{i}$. This approach closely mimics 
an explicit filtered LES method and allows us to examine the performance of RDM as applied to reacting scalars. The performance of RDM for implicitly filtered LES and subgrid reconstruction will be addressed in our future research. In addition, we solve the reactive scalars and temperature in conservative form to avoid modeling the commutation errors between the time derivative and the Favre filtering. A discussion of issues associated with commutation between time derivative and Favre filtering is provided in Appendix A. With this approach, the only source of error arises from applying RDM. The turbulent scalar mixing terms $\sigma_{\phi}$ and the chemical source terms are computed using the RDM closure. Equations $(7 \mathrm{c})$ and $(7 \mathrm{~d})$ are incorporated using explicit filtering with $\Delta / \delta_{s}=\{5.0,10.0\}$. These filter widths are comparable to those used in combustion LES applications. Initial conditions in the simulations were obtained by filtering the initial DNS scalar fields.

Because we use a low-Mach solver to conduct these simulations, the density is evaluated from the state equation. In addition, we treat $\mathrm{N}_{2}$ as an inert species in the simulation; hence $\rho Y_{\mathrm{N}_{2}}$ is evaluated from species mass balance. Therefore, density is derived from the local mass conservation and the equation of state, which takes the following form:

$$
\rho=\frac{\sum_{k}^{N_{s}-1}\left(\rho Y_{k}\right)^{\star}\left(1 / W_{k}-1 / W_{\mathrm{N}_{2}}\right)}{1 / \bar{W}-1 / W_{\mathrm{N}_{2}}},
$$

where $W_{k}$ is the molecular mass of species $k$ and $\bar{W}$ is the mean molecular mass of the gas mixture.

Figure 19 shows comparisons of the CO mass fraction field obtained from the explicitly filtered LES with RDM and the CO mass fraction field obtained from DNS, when $t / \tau_{u}=\{0.6,1.9,3.1,5.0\}$. The results presented here for the explicitly filtered LES are the deconvolved LES solutions in the post-processing step using RDM. We see from Fig. 19 that the CO mass fraction fields obtained from explicitly filtered LES for both filter widths qualitatively match DNS at each time instance. The high-wavenumber structures are reconstructed with high accuracy. At $t / \tau=0.6$, results for both $\Delta / \delta_{s}=\{5.0,10.0\}$ match DNS, as shown in the first column. As time evolves, we observe small discrepancies between the explicitly filtered LES results and DNS, particularly for highly localized structures. These discrepancies are more obvious for cases with $\Delta / \delta_{s}=10.0$ than for cases with $\Delta / \delta_{s}=5.0$. This indicates a deterioration of the performance of RDM as the filter width increases. This is expected because the damping of the signal increases as the filter width increases, which raises the difficulty of deconvolution. Nevertheless, the results from explicitly filtered LES using RDM show good agreement with DNS as time evolves.

Figures 20 show the temporal evolution of the mean and root-mean-square (RMS) fluctuations of $Y_{\mathrm{CH}_{4}}$, $Y_{\mathrm{CO}}, Y_{\mathrm{CO}_{2}}$ and $T$. In these figures, we see good agreement for all species between the explicitly filtered LES and DNS. This is consistent with the finding from the a priori analysis. The agreement in mean and variance profiles suggests that local discrepancies in scalar structure do not influence the statistical behavior of RDM. This is because the regularization enforced in RDM ensures the conservation of species both locally and globally.

Figure 21 shows the temporal evolution of mean chemical source terms of $\mathrm{CH}_{4}, \mathrm{CO}$ and $\mathrm{CO}_{2}$. We observe good agreement in chemical source terms for $\mathrm{CH}_{4}$ and $\mathrm{CO}_{2}$ over eight eddy-turnover times. Some differences in $\rho \dot{\omega}_{Y_{\mathrm{CO}}}$ are observed for $t / \tau_{u} \geq 4$. The mismatch between the explicitly filtered LES and DNS in the chemical source term of $\mathrm{CO}$ corresponds to conditions where the mean mass fraction of CO is small. Therefore the dynamics in the simulation are not significantly affected by this misprediction.

The results shown in this section illustrate that RDM applied as closure of the turbulence scalar mixing and source terms is stable with respect to time evolution. The statistics of reactive scalars and temperature are well captured and turbulent combustion is well predicted.

\section{Conclusions}

In this paper, a regularized deconvolution method (RDM) for reactive scalars is proposed. This method improves the accuracy of scalar reconstruction compared to the approximate deconvolution method (ADM) approach, and addresses drawbacks of existing linear deconvolution methods by ensuring boundedness and 


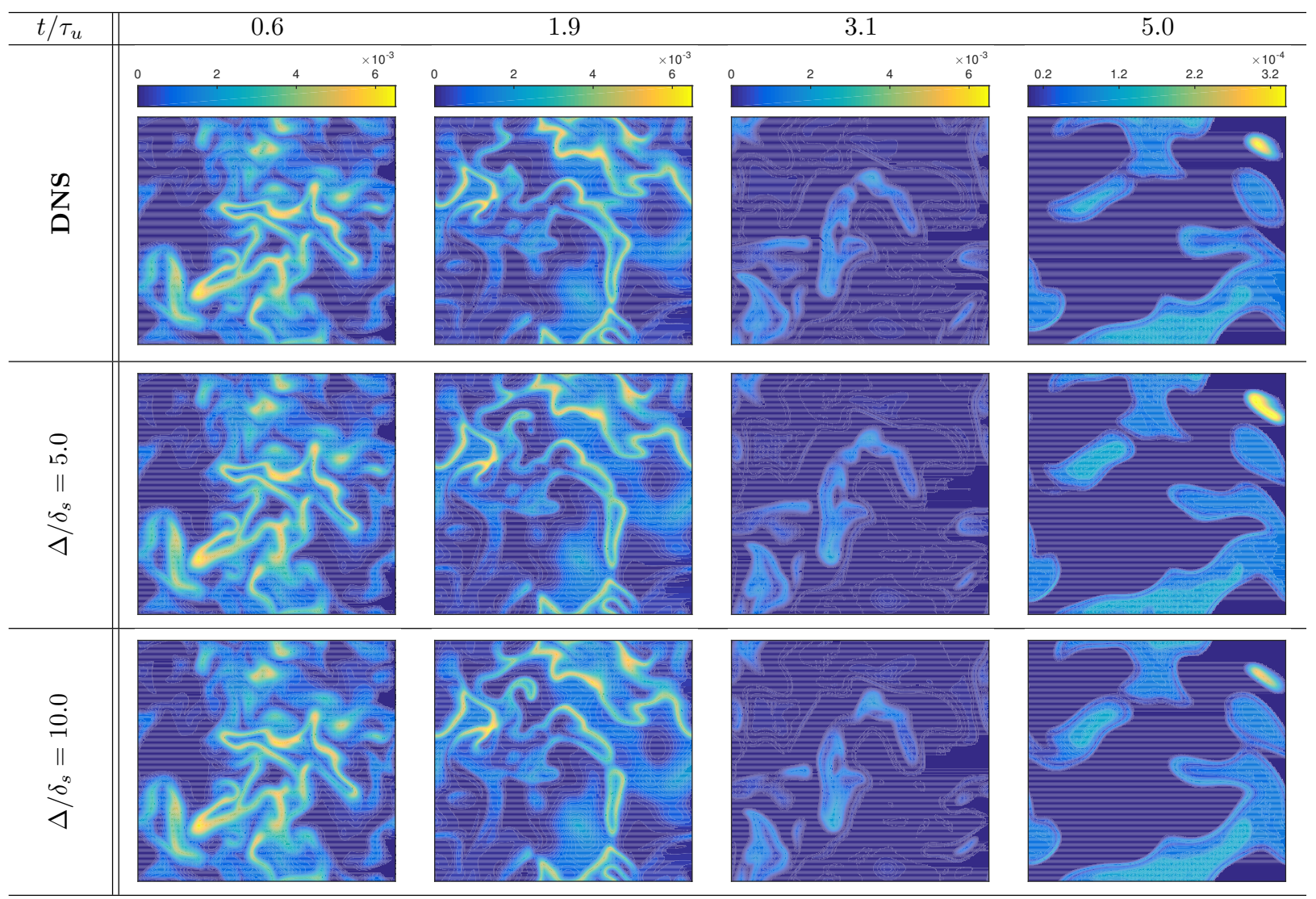

Figure 19: (Color online) CO mass fraction field obtained from DNS and explicitly filtered LES with RDM for $\Delta / \delta_{s}=\{5.0,10.0\}$ at $t / \tau_{u}=\{0.6,1.9,3.1,5.0\}$.

conservation of scalars. RDM combines a Wiener filter with a constrained minimum mean square error optimization problem to ensure conservation and boundedness of deconvoluted reactive scalars.

Both a priori and a posteriori analyses were conducted to examine the performance of RDM by considering a partially premixed flame configuration. Good accuracy and stable performance was obtained for scalar reconstruction using RDM with respect to filter width. The filtered chemical source terms and scalars that were correctly predicted from RDM are shown to preserve the flame topology.

By considering $\mathrm{CO}$ as a minor species in this study, the capability of RDM in handling scalars that have small magnitude but are critical to the reaction were illustrated. Additionally, computing the chemical source term in Arrhenius form assessed the capability of RDM in addressing high-order non-linearities. These properties were shown to be independent of the form of the chemistry model. Hence, RDM potentially extends the utilization of the deconvolution methods as closure model for turbulent combustion. For example, RDM overcomes shortcomings of presumed PDF methods by eliminating the need to make assumptions about combustion regimes.

We acknowledge that the a priori and a posteriori analyses do not consider all effects of LES. Some open issues, for example, the reconstruction of scales that are not represented on the coarse mesh, require investigation for application to implicit LES. These issues will be addressed in future work.

\section{Acknowledgment}

Support through the Stanford Graduate Fellowship and NASA with award number NNX15AV04A is gratefully acknowledged. Resources supporting this work were provided by the NASA High-End Computing 


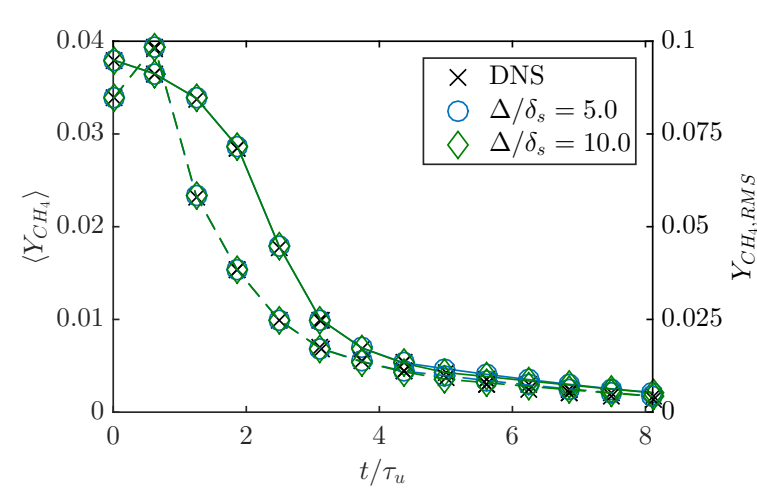

(a) $\mathrm{CH}_{4}$ mass fraction

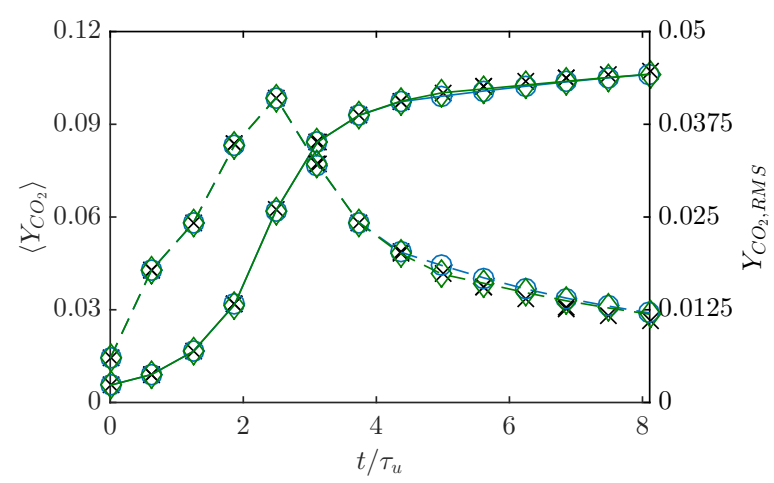

(c) $\mathrm{CO}_{2}$ mass fraction

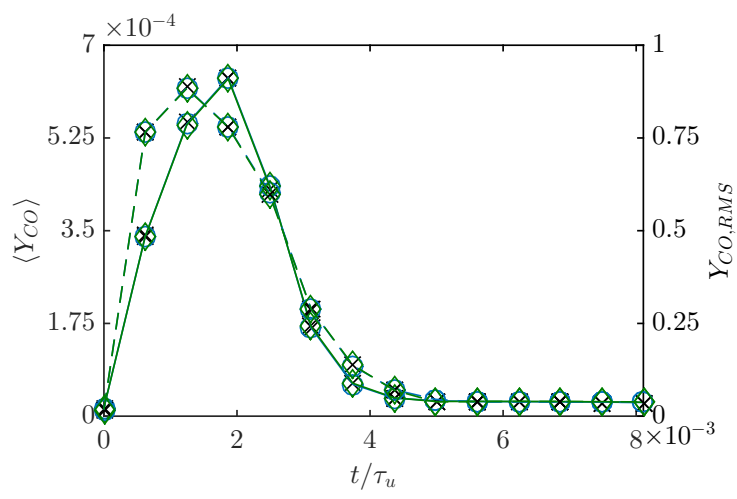

(b) $\mathrm{CO}$ mass fraction

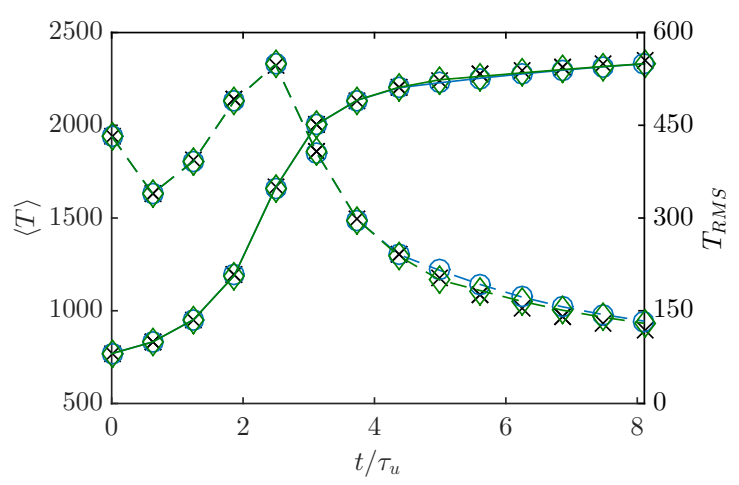

(d) Temperature

Figure 20: (Color online) Temporal evolution of mean (solid lines) and RMS (dashed lines) for $\mathrm{CH}_{4}, \mathrm{CO}, \mathrm{CO}_{2}$ mass fractions and temperature in DNS and explicitly filtered LES using RDM.

(HEC) Program through the NASA Advanced Supercomputing (NAS) Division at Ames Research Center and the National Energy Research Scientific Computing Center, a DOE Office of Science User Facility supported by the Office of Science of the U.S. Department of Energy under Contract No. DE-AC02$05 \mathrm{CH} 11231$.

\section{Appendix A. Commutation between deconvolution and time derivative}

In this appendix, an analysis is performed to the Favre deconvolution and filtering procedures. By rearranging Eq. (1) and denoting the right-hand-side terms as $\Psi$, we have

$$
\frac{\partial \rho \phi}{\partial t}=-\frac{\partial}{\partial x_{i}}\left(\rho u_{i} \phi\right)+\frac{\partial}{\partial x_{i}}\left(\rho D \frac{\partial}{\partial x_{i}} \phi\right)+\dot{\omega}(\phi) \equiv \Psi
$$

Without loss of generality, we write the semi-discretized form of Eq. (A.1) as:

$$
(\rho \phi)^{n}-(\rho \phi)^{n-1}=\Delta t \Psi\left(\phi^{n-1}\right)
$$

where the superscript denotes the time step. Define the Favre filter $\widetilde{G}$ of scalar $\phi$ at $t=t_{n}$ using density at $t=t_{n+1}$ as $\widetilde{G}^{n+1} \phi^{n}=\overline{(\rho \phi)^{n}} / \overline{\rho^{n+1}}$. Applying Favre filtering to Eq. (A.2) using density at $t=t_{n}$ and 


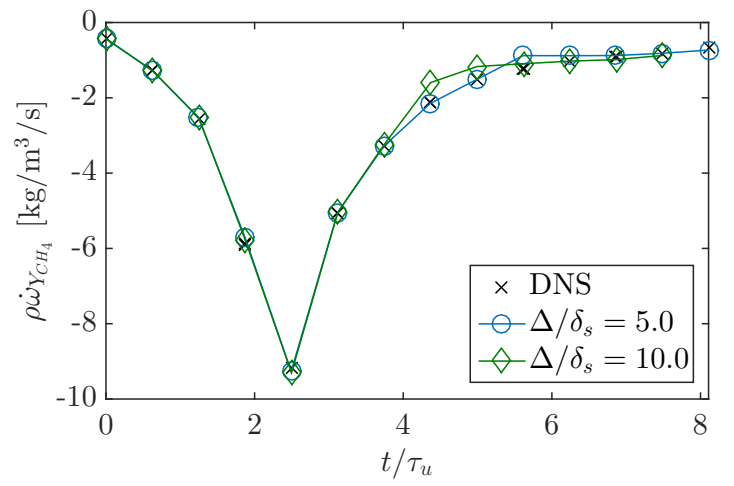

(a) $\mathrm{CH}_{4}$ source term

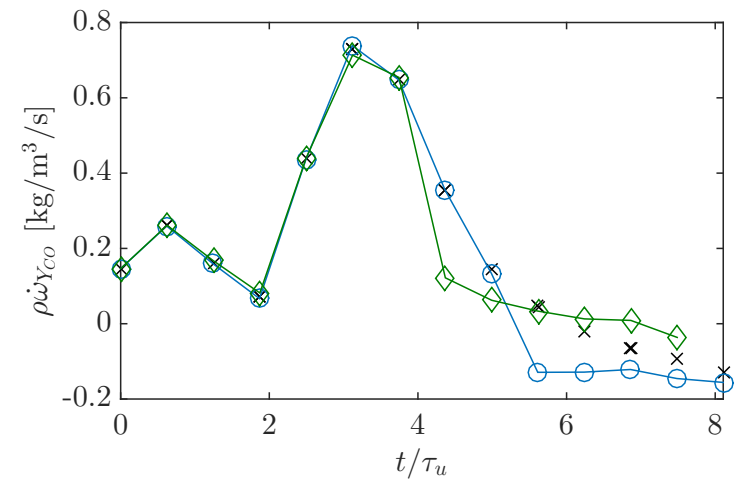

(b) $\mathrm{CO}$ source term

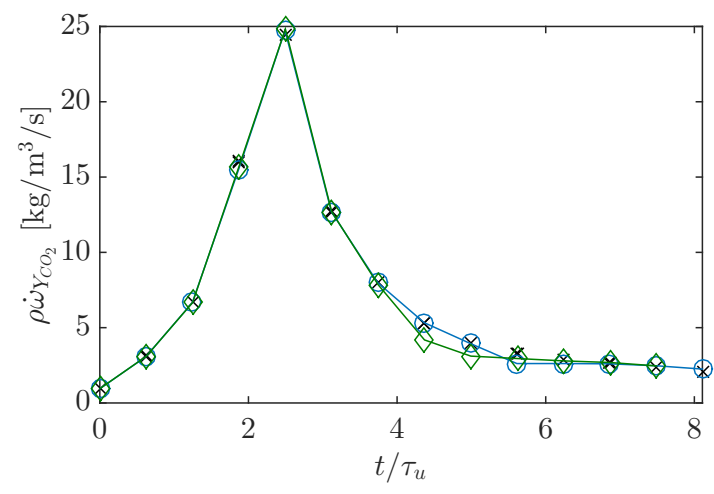

(c) $\mathrm{CO}_{2}$ source term

Figure 21: (Color online) Mean chemical source term profile for $\mathrm{CH}_{4}, \mathrm{CO}, \mathrm{CO}_{2}$ and temperature in DNS and explicitly filtered LES using RDM.

$t=t_{n-1}$ respectively, we obtain the following set of equations:

$$
\begin{aligned}
\widetilde{G}^{n} \phi^{n}-\widetilde{G}^{n} \phi^{n-1} & =\Delta t\left(\widetilde{G}^{n} \Psi^{n-1}\right)=\widetilde{\phi}^{n}-\widetilde{G}^{n} \phi^{n-1}, \\
\widetilde{G}^{n-1} \phi^{n}-\widetilde{G}^{n-1} \phi^{n-1} & =\Delta t\left(\widetilde{G}^{n-1} \Psi^{n-1}\right)=\widetilde{G}^{n-1} \phi^{n}-\widetilde{\phi}^{n-1} .
\end{aligned}
$$

By adding Eqs. (A.3) and rearrange terms, the following equation is obtained:

$$
\left(\widetilde{G}^{n}+\widetilde{G}^{n-1}\right) \phi^{n}-\left(\widetilde{G}^{n}+\widetilde{G}^{n-1}\right) \phi^{n-1}=\Delta t\left(\widetilde{G}^{n}+\widetilde{G}^{n-1}\right) \Psi^{n-1} .
$$

From this equation, it can be seen that the Favre filter operation does not commute with the temporal derivative. Hence, the Favre filtering has to be done implicitly if the primitive variables are solved for. This could be done iteratively but will induce additional cost. This issue is avoided if conservative variables are solved for, which can be expressed by the following time-discretized filtered equation:

$$
\overline{(\rho \phi)}^{n}-\overline{(\rho \phi)}^{n-1}=\Delta t \overline{\Psi\left(\phi^{n-1}\right)}
$$

where the filter operation is no longer a function of $\rho$. In this approach, deconvolved scalars can be evaluated as $\phi^{\star}=Q *(\bar{\rho} \widetilde{\phi}) / \rho$, where $\rho$ is computed at the current time step from Eq. (40) using the deconvolved conserved scalars without ambiguity. 
[1] H. Pitsch. Large-eddy simulation of turbulent combustion. Annu. Rev. Fluid Mech., 38(1):453-482, 2006.

[2] S. B. Pope. Ten questions concerning the large-eddy simulation of turbulent flows. New J. Phys., 6:1-24, 2006.

[3] M. Boger, D. Veynante, H. Boughanem, and A. Trouvé. Direct numerical simulation analysis of flame surface density concept for large eddy simulation of turbulent premixed combustion. Proc. Combust. Inst., 27(1):917-925, 1998.

[4] P. Moin, K. Squires, W. Cabot, and S. Lee. A dynamic subgrid-scale model for compressible turbulence and scalar transport. Phys. Fluids, 3(11):2746-2757, 1991.

[5] O. Colin, F. Ducros, D. Veynante, and T. Poinsot. A thickened flame model for large eddy simulations of turbulent premixed combustion. Phys. Fluids, 12(7):1843-1863, 2000.

[6] A. R. Kerstein. A linear-eddy model of turbulent scalar transport and mixing. Combust. Sci. Tech., 60(4-6):391-421, 1988.

[7] U. Maas and S. B. Pope. Simplifying chemical kinetics: intrinsic low-dimensional manifolds in composition space. Combust. Flame, 88:239-264, 1992.

[8] O. Gicquel, N. Darabiha, and T. Dominique. Laminar premixed hydrogen/air counterflow flame simulations using flame prologation of ILDM with differential diffusion. Proc. Combust. Inst., 28:1901-1908, 2000.

[9] J. Oijen and L. Goey. Modelling of premixed laminar flames using flamelet generated manifolds. Combust. Sci. Tech., 161(1):113-137, 2000.

[10] C. D. Pierce and P. Moin. Progress-variable approach for large-eddy simulation of non-premixed turbulent combustion. J. Fluid Mech., 504:73-97, 2004.

[11] H. Wu, Y. C. See, Q. Wang, and M. Ihme. A Pareto-efficient combustion framework with submodel assignment for predicting complex flame configurations. Combust. Flame, 162(11):4208-4230, 2015.

[12] H. Wu and M. Ihme. Compliance of combustion models for turbulent reacting flow simulations. Fuel, 2016. in press

[13] A. Potturi and J. Edwards. Investigation of subgrid closure models for finite-rate scramjet combustion. AIAA Paper 2013-2461, 2013.

[14] E. Giacomazzi, C. Bruno, and B. Favini. Fractal modelling of turbulent combustion. Combust. Theor. Model., 4(4):391$412,2000$.

[15] K. Nogenmyr, C. Fureby, X. Bai, P. Petersson, R. Collin, and M. Linne. Large eddy simulation and laser diagnostic studies on a low swirl stratified premixed flame. Combust. Flame, 156(1):25-36, 2009.

[16] P. DesJardin and S. Frankel. Large eddy simulation of a nonpremixed reacting jet: Application and assessment of subgridscale combustion models. Phys. Fluids, 10(9):2298-2314, 1998.

[17] B. Fiorina, R. Vicquelin, P. Auzillon, N. Darabiha, O. Gicquel, and D. Veynante. A filtered tabulated chemistry model for LES of premixed combustion. Combust. Flame, 157(3):465-475, 2009.

[18] K. N. C. Bray, M. Champion, P. A. Libby, and N. Swaminathan. Finite rate chemistry and presumed PDF models for premixed turbulent combustion. Combust. Flame, 146(4):665-673, 2006.

[19] S. Stolz and N. A. Adams. An approximate deconvolution procedure for large-eddy simulation. Phys. Fluids, 11:1699-1701, 1999.

[20] S. Stolz, N. A. Adams, and L. Kleiser. The approximate deconvolution model for large-eddy simulations of compressible flows and its application to shock-turbulent-boundary-layer interaction. Phys. Fluids, 13(10):2985-3001, 2001.

[21] S. Stolz, N. A. Adams, and L. Kleiser. An approximate deconvolution model for large-eddy simulation with application to incompressible wall-bounded flows. Phys. Fluids, 13(4):997-1015, 2001.

[22] P. Schlatter, S. Stolz, and L. Kleiser. LES of transitional flows using the approximate deconvolution model. Int. J. Heat Fluid Fl., 25(3):549-558, 2004.

[23] C. Pantano and S. Sarkar. A subgrid model for nonlinear functions of a scalar. Phys. Fluids, 13(12):3803-3819, 2001.

[24] J. Mellado, S. Sarkar, and C. Pantano. Reconstruction subgrid models for nonpremixed combustion. Phys. Fluids, 15(11):3280-3307, 2003.

[25] J. Oefelein. Large eddy simulation of turbulent combustion processes in propulsion and power systems. Prog. Aerospace Sci., 42(1):2-37, 2006.

[26] P. Domingo and L. Vervisch. Large eddy simulation of premixed turbulent combustion using approximate deconvolution and explicit flame filtering. Proc. Combust. Inst., 35(2):1349-1357, 2015.

[27] B. Müller. Low-Mach-number expansion of the Navier-Stokes equations. J. Eng. Math., 34(1-2):97-109, 1998.

[28] W. Layton and L Rebholz. Approximate Deconvolution Models of Turbulence: Analysis, Phenomenology and Numerical Analysis. Springer, Berlin Heidelberg, 2012.

[29] N. Wiener. Extrapolation, interpolation, and smoothing of stationary time series. The MIT press, Cambridge, MA, 1949.

[30] J. Nocedal and S. Wright. Numerical optimization. Springer Verlag, Berlin, New York, 2006.

[31] F. Dryer and I. Glassman. High-temperature oxidation of CO and $\mathrm{CH}_{4}$. Proc. Combust. Inst., 14(1):987-1003, 1973.

[32] C. Westbrook and F. Dryer. Simplified reaction mechanisms for the oxidation of hydrocarbon fuels in flames. Combust. Sci. Tech., 27(1-2):31-43, 1971.

[33] M. Ihme and Y. C. See. LES flamelet modeling of a three-stream MILD combustor: Analysis of flame sensitivity to scalar inflow conditions. Proc. Combust. Inst., 33(1):1309-1317, 2011.

[34] H. Yamashita, M. Shimada, and T. Takeno. A numerical study on flame stability at the transition point of jet diffusion flames. Proc. Combust. Inst., 26(1):27-34, 1996. 